\title{
Interne Effizienz von Bildungssystemen
}

\section{Dieter Dohmen}

Der Beitrag untersucht die wesentlichen Bestimmungsfaktoren für die Effizienz von Bildungssystemen und Möglichkeiten zu deren Verbesserung. Ausgehend von einer Analyse der individuellen Interessen der am Bildungssystem unmittelbar beteiligten Personengruppen werden auf drei Ebenen die Zusammenhänge untersucht, die die Effizienz von Bildungssystemen determinieren können. Hierzu zählen Inputund Outputfaktoren im Sinne einer Bildungsproduktionsfunktion, organisatorische Rahmenbedingungen und neue Finanzierungsmodelle, die auf eine stärkere Ausrichtung auf Wettbewerb und Markt setzen.

Betrachtet man die vorliegende empirische Evidenz, dann scheint eine ausschliessliche Ressourcenerhöhung wenig Einfluss auf Schülerleistungen zu haben. Erfolgversprechender dürften Reallokationen der vorhandenen Ressourcen zugunsten von Sachmitteln oder die Einführung eines leistungsorientierten Vergütungssystems, vor allen Dingen aber strukturelle Massnahmen sein. So sind etwa zentrale Prüfungsverfahren mit signifikant besseren Schülerleistungen verbunden. Gleiches gilt auch für dezentrale Prozess- und Personalverantwortung. Die Übertragung der vollständigen Budgetverantwortung auf die Bildungseinrichtungen führt jedoch eher zu schlechteren Schülerleistungen, da Ressourcen vor allen Dingen zugunsten der Lehrerinteressen eingesetzt werden.

Höchst umstritten sind die Effekte von Bildungsgutscheinen und anderen wettbewerbsfördernden Finanzierungsinstrumenten. Betrachtet man die Ergebnisse von Evaluationen entsprechender Modelle, dann ist die Leistungssteigerung bei den Schülern weniger ausgeprägt als viele Befürworter behaupten. Allerdings kommen die meisten Studien zu einem leicht positiven Zusammenhang, der durch weitergehende strukturelle Reformen verbessert werden könnte. Hierzu zählen die strukturellen Rahmenbedingungen ebenso wie modellimmanente Veränderungen (differenziertere Fördermechanismen) sowie insbesondere Beratung und Information. Durch eine solchermassen ausgestaltete und im übergreifenden Zusammenhang strukturierte Reform werden die Anreize für alle Beteiligten in eine leistungssteigernde Richtung modifiziert. Werden nur Teillösungen realisiert, dann ist zu erwarten, dass die Effizienzsteigerungen weniger stark sind. 


\section{Einleitung}

Die Frage nach der Effizienz von Bildungssystemen hat in den letzten Jahren, nicht zuletzt durch die internationalen Leistungsvergleiche (TIMSS und PISA) einen erheblichen Bedeutungszuwachs erlangt. Darüber hinaus sind aber auch andere Gründe wie knappe öffentliche Ressourcen und dauerhafte Forderungen nach «mehr Geld für Bildung» zu nennen. Einerseits stehen Bildungsausgaben im politischen Verteilungskampf mit anderen Bereichen und andererseits ist ein Ressourcenzuwachs nur vertretbar, wenn die bereits vorhandenen finanziellen Mittel effizient genutzt werden. Andernfalls könnte eine Reallokation der vorhandenen Mittel zu besseren Schülerleistungen oder gar Einsparungen führen. Damit ist der Kern, der im Folgenden zu untersuchenden Fragestellung genannt: Werden die vorhandenen Ressourcen effizient ${ }^{1}$ eingesetzt und welche sind die Bestimmungsfaktoren für einen solchen effizienten Ressourceneinsatz. Ferner ist zu klären, was der Erfolgsmassstab für ein solches effizientes Bildungssystem ist.

Davon ausgehend, dass der Erfolg bzw. die Effizienz des Bildungssystems von unterschiedlichen Akteuren unterschiedlich definiert und betrachtet werden kann, wird zunächst untersucht, welche Akteure von Bedeutung sind und welche Interessen sie verfolgen. Dies erfolgt auf der Grundlage einer PrincipalAgent-Theorie, die davon ausgeht, dass alle Akteure und deren Vertreter vor allen Dingen ihre eigenen Interessen bzw. die der Gruppe verfolgen.

In einem zweiten Schritt wird dann anhand verschiedener Inputfaktoren untersucht, welcher Zusammenhang zwischen den eingesetzten finanziellen Ressourcen und den Schülerleistungen besteht. Liesse sich beispielsweise zeigen, dass höhere Ausgaben mit besseren Schülerleistungen verbunden sind, könnte dies eine Unterstützung für die Forderung nach mehr Geld sein, vorausgesetzt, durch eine Umverteilung der Mittel wäre nicht ebenfalls eine Verbesserung zu erreichen.

Anschliessend wird auf einer weiteren Ebene untersucht, inwieweit bestimmte strukturelle Rahmenbedingungen (Zentralexamina, eingliedrige Bildungssysteme, Schulautonomie) systematisch mit besseren Schülerleistungen verbunden sind.

Auf einer letzten Ebene wird dann der Frage nachgegangen, inwieweit eine Neuordnung der Bildungsfinanzierung, die häufig unter divergierenden Überschriften geführt wird, u.a. privat versus öffentlich, Gutscheine contra staatlicher Finanzierung oder Wettbewerb zu einer Steigerung der Leistungsfähigkeit des Bildungssystems beitragen können.

Letztlich fasst der vorliegende Artikel damit drei Ebenen zusammen und geht der Frage nach, ob sich systematische Zusammenhänge zwischen Ressourcen, Organisationsstrukturen und Finanzierungsmodellen identifizieren lassen, die zu einem leistungsstärkeren Bildungssystem führen können. 


\section{Bildungspolitik im Widerstreit der verschiedenen Interessen}

Im Bildungssystem sind mehrere Akteure vertreten, die jeweils ihre eigenen Interessen verfolgen, um ihren persönlichen Nutzen zu maximieren. Schülerinnen und Schüler, Eltern, Lehrerinnen und Lehrer, Administratoren auf schulischer und ministerieller Ebene sowie Politiker (Carnoy, 1995a; Wössmann 2002).

Der ökonomischen Theorie folgend wollen Schülerinnen und Schüler einen möglichst guten Schulabschluss erreichen, wozu sie neben den schulischen Inputfaktoren auch ihre eigene Freizeit sowie andere Ressourcen (Bücher, Fernsehen, PCs etc.) nutzen. Ihr familiärer, sozioökonomischer Hintergrund ist dabei ebenso eine externe Variable wie eventuelle Behinderungen oder sonstige Benachteiligungen oder Fähigkeiten. ${ }^{2}$ In Abhängigkeit von zentralen oder dezentralen Leistungsstandards ist anzunehmen, dass sie sich unterschiedlich stark anstrengen werden. So haben sie bei zentralen Leistungsüberprüfungen kaum einen Anreiz, das Leistungsniveau in der Klasse abzusenken, da sie hiervon keinen Vorteil haben, wenn dies in anderen Schulen bzw. Klassen nicht der Fall ist. Erfolgt aber die Leistungsbestimmung anhand des relativen Leistungsvermögens innerhalb der jeweiligen Klasse, dann besteht tendenziell ein Anreiz, hierauf (negativen) Einfluss zu nehmen, was vor allen Dingen für leistungsschwächere oder unmotivierte Schüler gelten dürfte.

In dem Bestreben nach einem guten Schulabschluss werden sie von altruistischen Eltern unterstützt, da die Nutzenfunktion der Kinder Bestandteil ihrer eigenen Nutzenmaximierung ist. Sie vertreten dann soweit möglich die oben genannten Interessen der Kinder. Handelt es sich um egoistische oder nicht ausschließlich altruistische Eltern, dann werden sie nicht nur die Interessen der Kinder, sondern auch ihre eigenen verfolgen. Auch wenn dies eine Einschränkung hinsichtlich des Verfolgungsgrades der Interessen des Kindes bedeutet, kann davon ausgegangen werden, dass sie ein Interesse an einem möglichst guten Schulabschluss der Kinder haben.

Eltern und Schüler werden daher - unter Berücksichtigung der finanziellen und anderer Beschränkungen (Entfernung zur Schule, Schulangebot, Qualität der Lehrkräfte, Schulgebühren etc.) - die Schule auswählen, die ihren Vorstellungen am besten entspricht. Dies dürfte ceteris paribus eher eine Schule mit kleinen Klassen, guten Lehrern und Schülern bzw. einer guten Sozialstruktur sein.

Lehrerinnen und Lehrer verfolgen demgegenüber ihre eigenen Interessen. Wie ihre Nutzenmaximierung konkret aussieht, ist in starkem Masse von den vorhandenen Anreizmechanismen abhängig. Wichtige Faktoren sind sicherlich ein gutes Einkommen, gesellschaftliche Anerkennung, Beamtenstatus, Unkündbarkeit sowie relativ grosszügige Ferienregelungen. In den meisten Fällen dürfte auch die Performance der Schüler Bestandteil der Nutzenfunktion der Lehrer sein. 
Hinsichtlich der Unterrichtsgestaltung sind zunächst ihre Ausbildung und individuellen Fähigkeiten etc. als externe Variablen von Interesse. Welchen Aufwand sie zur Vorbereitung des Unterrichts betreiben, dürfte ceteris paribus von den externen Anreizen und der intrinsischen Motivation abhängig sein. Ist beispielsweise das Gehalt fixiert und nicht von Leistung oder anderen Faktoren abhängig, dann werden sie tendenziell weniger Aufwand zur Unterrichtsvorbereitung betreiben, als wenn sie an bestimmten Leistungsstandards gemessen und deren Erfüllungs-grad kontrolliert werden. Es ist ferner anzunehmen, dass sie ihren Zeiteinsatz auSSerhalb des Unterrichts minimieren werden, und die Unterrichtsgestaltung wird ihren unmittelbaren Interessen und weniger denen der Schüler folgen. ${ }^{3}$

Dies kann dazu führen, dass sie die Ressourcen einsetzen, die ihren eigenen Nutzen maximieren, unabhängig davon, ob sie den Unterricht und damit das Leistungsniveau der Schüler verbessern. Soweit sie Einfluss auf die Klassenzusammensetzung haben, wäre eine naheliegende Folge, dass sie kleine Klassen mit sehr guten Schülern bevorzugen würden.

In diesem Punkt gibt es somit wahrscheinlich eine Interessensidentität von Schülern, Eltern und Lehrern, unabhängig davon, ob kleinere Klassen wirklich (in jedem Fall) zu besseren Schülerleistungen führen.

Haben die Lehrer Einfluss auf die Ressourcenverwendung, dann ist zu erwarten, dass sie diese entsprechend ihrer eigenen Nutzenfunktion einsetzen, d.h. sie werden tendenziell eher ihr Gehalt erhöhen als zusätzliche Sachmittel beschaffen. Auf übergeordneter Ebene werden ihre Interessen von Gewerkschaften vertreten. Auch diese werden daher eher eine Ressourcenallokation zugunsten der Lehrer fordern, woraus üblicherweise die Forderung nach höheren Gehältern resultieren wird.

Zumindest theoretisch spricht demzufolge einiges dafür, dass die Existenz externer Anreizmechanismen wie etwa leistungsbezogene Gehälter und Zentralexamina zu besserer Unterrichtsqualität und damit - indirekt - zu besseren Schülerleistungen führen werden. Als vergleichbare Kontrollmechanismen bzw. externe Anreize werden auch eine wettbewerbliche Verfassung des Bildungssystems oder marktorientiertere Finanzierungsinstrumente diskutiert. Wenn die Nachfrager sich an der Qualität der Bildungseinrichtungen orientieren und sich somit rational verhalten, dann werden sie bessere Schulen auswählen.

Die Maximierungsfunktion der Schuladministration hängt ebenfalls von den vorhandenen Anreizmechanismen ab. Besteht z.B. ein Zusammenhang zwischen der Anzahl der Schüler und dem Schulbudget, dann sie an einer grossen Schülerzahl interessiert sein. D.h., wenn Eltern und Schüler sich an der Leistungsfähigkeit der Schule orientieren, würde sie versuchen, einer möglichst guten Schule mit guten Schülerleistungen vorzustehen.

Ist das Budget aber gegeben, dann wird der Schuladministration die Schülerzahl eher egal sein; vielleicht besteht sogar ein Interesse, möglichst wenig Schüler zu haben, um weniger Arbeit und mehr Freizeit zu haben. Dies dürfte um so 
mehr gelten, wenn zugleich das eigene Gehalt und die zukünftige Berufslaufbahn mehr oder weniger determiniert. Zu erwarten ist in diesem Fall auch, dass sie es sich möglichst nicht mit den Lehrern, mit denen sie täglich zu tun hat, «verscherzen» wird, d.h. sie vor allem ihren Forderungen nachgeben. Es könnte somit zu einer «unheiligen» Interessenallianz kommen, die einseitig den Interessen der Lehrer entspricht.

Dies dürfte teilweise gleichermassen für Administratoren auf Schul- wie auf Ministeriumsebene gelten, wobei letztere bei der Mittelzuweisung auf die einzelnen Schulen evtl. dadurch eingeschränkt sind, dass sie mit dem vorgesehenen Gesamtbudget auskommen müssen. Meist werden dabei, um keinen Konflikt zu provozieren, die frei aufteilbaren Mittel tendenziell gleichmässig auf alle Schulen verteilt, da sich dann keiner benachteiligt fühlen kann. Der Bürokratie-Theorie folgend, werden die Administratoren - ihren eigenen Interessen folgend - für eine ausufernde Bürokratie sorgen.

Politiker sind vor allen Dingen an ihrer Wiederwahl interessiert und werden daher die Politik verfolgen, die ihnen dabei am Erfolg versprechensten erscheint. Dies bedeutet, dass die relative Marktmacht der verschiedenen Interessengruppen und Akteure von entscheidender Bedeutung ist. Dies sei an einem kurzen Beispiel illustriert. Nehmen wir an, es gibt eine «Eliteschule», auf die vor allen Dingen die Kinder aus sozioökonomisch starken Familien gehen, während die «Restschule» alle andere Schüler aufzunehmen hat. Wenn die erstgenannte Gruppe politisch relativ viel Marktmacht entfalten kann, was durchaus wahrscheinlich ist, dann wird die «Eliteschule» relativ mehr Mittel erhalten als die «Restschule», unabhängig davon, wo diese Ressourcen eventuell besser eingesetzt werden können.

Allerdings ist dabei zu berücksichtigen, dass die Politiker nicht nur die Interessen der unmittelbar bildungsrelevanten Interessengruppen in ihr Maximierungsverhalten einbeziehen werden, sondern auch die Interessen anderer Gruppen, z.B. der Wirtschaft. Dies bedeutet, dass hier ein Korrektiv für einseitige Massnahmen zugunsten bestimmter Bildungsgruppen bestehen könnte. So könnte z.B. die Klage der Wirtschaft nach unzureichend qualifizierten Absolventen des Schulsystems dann ein höheres Gewicht erhalten, wenn sie neben dem Hinweis auf die damit verbundenen wirtschaftlichen Konsequenzen (niedrigeres Wachstum, Verlust oder Einschränkung der Wettbewerbsfähigkeit, höhere Arbeitslosigkeit etc.) auch eine hohe Marktmacht im Verteilungskampf der Interessen in die Wagschale werfen können. ${ }^{4}$ Diese konkurrierenden Interessensverbände könnten somit über den Umweg der Politikbeeinflussung ein Korrektiv zu den Forderungen der Lehrergewerkschaften sein.

Die vorstehenden Ausführungen bilden den Rahmen für die Darstellungen in den folgenden Abschnitten. Anhand von empirischen Arbeiten wird der genannte theoretische Ansatz überprüft, verfeinert, modifiziert oder verworfen. Begonnen sei mit dem mikroökonomischen Ansatz, der der Frage nachgeht, welcher Zusammenhang zwischen den Inputfaktoren und den Schülerleistungen besteht. 


\section{Die Bedeutung unmittelbarer Inputfaktoren und indikatoren auf Schülerleistungen - Bildungs- produktionsfunktion}

Seit dem Erscheinen des Coleman-Reportes «Equality of Educational Opportunity» (Coleman et al., 1966) gibt es in der Fachliteratur, aber auch in der Politik, eine anhaltende Diskussion über die Frage, ob zusätzliche Ressourcen an Schulen zu einer Verbesserung der Schülerleistungen führen. Gerade im Nach-PISADeutschland wird die Forderung nach mehr Geld für Bildung lauter. Lässt sich damit automatisch die Qualität des Bildungswesens verbessern?

Die Erhöhung der finanziellen Ressourcen kann über verschiedene Kanäle zu einer Verbesserung der Schülerleistungen führen. Wir werden diese Überlegung anhand einer relativ einfachen Bildungsproduktionsfunktion untersuchen. In Anlehnung an Hanushek ist die Schülerleistung abhängig vom familiären Hintergrund, von den Lehrern und sonstigen schulischen Inputfaktoren.

Aus Sicht der Bildungseinrichtung bzw. des Bildungssystem ist der familiäre Hintergrund der Schülerinnen und Schüler eine weitgehend externe Variable, wobei Studien immer wieder auf die zentrale Bedeutung des sozioökonomischen Hintergrundes verweisen (u.a. Coleman et al., 1966, OECD, 2001). 5 Teilweise wird deshalb auch von einer gemeinsamen Bildungsproduktion von Familie und Schule gesprochen (Carnoy, 1995b).

Der Faktor Lehrer ist quantitativ und qualitativ von Bedeutung. Quantitativ wird dies in der Regel an der Schüler-Lehrer-Relation gemessen, die eine abgeleitete Grösse aus Unterrichtsstunden der Lehrer (Kontaktzeit) ${ }^{6}$ und Instruktionszeit der Schüler ${ }^{7}$ und der eigentlichen Klassengrösse ist.

Die Qualität der Lehrkräfte bzw. des Unterrichts ist wesentlich schwieriger zu erfassen. Sie ist u.a. eine Funktion aus der Qualität der Lehrkraft (abhängig u.a. von deren Aus- und Fortbildung sowie Fähigkeit etc.) und der im Hinblick auf die Thematik angewandten Unterrichtsmethodik sowie der eingesetzten Lehrund Lernmittel. Aus Kostensicht ist einerseits das Gehalt der Lehrer von Bedeutung, das von unterschiedlichen Komponenten wie Einstiegsgehalt, Alter als Proxi für die Berufserfahrung, Familienstand und eventuellen Leistungszulagen abhängig ist. Andererseits spielen die Sachausgaben für Lehr- und Lernmittel, Raumausstattung sowie Investitionen eine wichtige Rolle.

Die Bildungskosten sind somit eine Funktion des Gehalts für Lehrkräfte und sonstiges Personal, der sonstigen laufenden Sachkosten und der Investitionen. Eine Ressourcenerhöhung kann somit z.B. für mehr oder bessere Lehrkräfte, für eine Erhöhung der Kontaktzeit der Lehrkräfte oder der Erhöhung der Instruktionszeit der Schüler, mehr Lehr- und Lernmittel oder Investitionen ausgegeben werden. Unter Gesichtspunkten der Kosten-Wirksamkeits-Analyse wäre dann zu fragen, durch welche Verwendung sich die größtmögliche Leistungsverbesserung ergeben würde. Die bisher am meisten untersuchte Korrelation ist die zwischen den Bildungsausgaben und der Schülerleistung 


\section{Bildungsausgaben allgeme in}

Insgesamt haben sich bereits relativ viele Untersuchungen mit der Frage beschäftigt, ob zusätzliche Ressourcen mit einer Leistungssteigerung bei den Schülern verbunden ist. Zusammenfassend kommen die meisten Studien allenfalls zu einem schwachen positiven Zusammenhang zwischen Bildungsausgaben und Schülerleistung (siehe u.a. Figlio, 1999; Gundlach/Wössmann, 2003, Wössmann, 2002), dies gilt auch in neueren Studien z.B. für Großbritannien (Levacic/Vignoles, 2002). ${ }^{8}$

In einer Zeitreihenanalyse fand Wössmann (2002) heraus, dass die moderate Erhöhung der realen Ausgaben je Schüler nur in Schweden und den Niederlanden einen positiven Einfluss auf die Schülerleistung über die Zeit hatte. Bis auf Italien war für alle anderen untersuchten OECD-Länder der Einfluss über die Zeit negativ, d.h. die Schülerleistungen stiegen nicht proportional zur Erhöhung der realen Ausgaben je Schüler. Daraus folgerte er, dass nur eine sehr geringe Evidenz die Annahme einer verbesserten Schülerleistung durch erhöhte Ausgaben im Zeitverlauf unterstütze und somit die Ausgaben je Schüler keine signifikante Determinante der Schülerleistungen seien. ${ }^{9}$

Somit ergibt sich allenfalls eine geringe Evidenz für die Annahme, dass mehr finanzielle Mittel auch zu besseren Schülerleistungen führen.

\section{Schüler-Lehrer-Relation}

Mit am meisten diskutiert und theoretisch plausibel erscheint eine Reduktion der Schüler-Lehrer-Relation, da dies eine bessere Betreuung der einzelnen Schüler ermöglichen würde. Aufgrund der erheblichen Bedeutung für die Bildungsausgaben ist dieser Zusammenhang auch empirisch immer wieder untersucht worden.

Fasst man die vorliegenden Untersuchungen zusammen, dann ergibt sich keine empirische Evidenz für eine generelle Reduktion der Schüler-Lehrer-Relation. Vielmehr kommen die vorliegenden Studien zu sehr unterschiedlichen Ergebnissen. So kommen einzelne Studien sogar zu einer positiven oder teilweise positiven Korrelation (Dohmen \& Haileselassie, 2003), allerdings auf der Grundlage aggregierter Vergleichsdaten, während andere Studien zu dem erwarteten negativen Zusammenhang kommen. Auch divergieren die Ergebnisse in Abhängigkeit von der Betrachtungsebene. D.h. Analysen auf der Grundlage internationale Vergleichuntersuchungen wie TIMSS und PISA kommen zu teilweise anderen Ergebnissen als Untersuchungen, die die Ursachen für Leistungsdifferenzen innerhalb einzelner Länder betrachten.

So kommen die meisten Untersuchungen auf der Grundlage von TIMSSoder PISA-Daten zu dem Ergebnis, dass es keine signifikanten Zusammenhänge zwischen der Klassengröße und den Schülerleistungen (siehe u.a. Wössmann, 2002, Gundlach/Wössmann, 2003), während Fertig (2002, 2003) bezogen auf Deutschland eine signifikante Korrelation in der üblicherweise erwarteten Richtung identifiziert, d.h. Schüler in kleineren Klassen weisen bessere Ergebnisse auf als Schüler in größeren Klassen. ${ }^{10}$ 
Neben den uneinheitlichen Ergebnissen, die sich auch aus früheren Arbeiten ableiten lassen (Hanushek, 1989; Hedges, Glass \& Smith 1979; Laine \& Greenwald, 1994, Odden 1990 sowie für Deutschland Ingenkamp, Petillon \& Weiss, 1985), weisen auch andere Überlegungen darauf hin, dass der Zusammenhang zwischen der Schüler-Lehrer-Relation von weitergehenden Faktoren beeinflusst werden dürfte. So können etwa Wahlentscheidungen der Eltern und Schüler dazu führen, dass Rückkopplungen bestehen, so z.B. dadurch, dass sie in Schulen angemeldet werden, die in einem besseren sozioökonomischen Umfeld liegen oder ihren Wohnsitz entsprechend wählen. Auch können zentrale Zuweisungsverfahren mit unterschiedlichen Klassengrössen in einzelnen Schulen oder innerhalb derselben Schule die Schülerleistungen beeinflussen (Gundlach \& Wössmann, 2003). Diese Überlegungen betonen den Aspekt eines lokalen öffentlichen Gutes, den Bildung bzw. Schülerleistungen im Rahmen eines Klassenverbandes hat, sie kann damit letztlich eine endogene Variable werden (Lazear, 2001). ${ }^{11}$

Dies kann zugleich ein Indiz dafür sein, dass unterschiedliche Rahmenfaktoren, wie etwa der sozioökonomische Hintergrund der Schüler oder individuelle Schülerfaktoren wie etwa Behinderungen oder Benachteiligungen sowie das Schülerverhalten, erheblichen Einfluss auf die "optimale» Klassengröße bzw. Schüler-Lehrer-Relation haben (Lazear, 2001). So können Klassen mit guten oder sich wohlverhaltenden Schülern grösser sein als Klassen, in denen Schüler sind, die stören oder Lernschwierigkeiten haben etc.

In diese Richtung weisen auch Studien, die unter bestimmten Bedingungen, z.B. für benachteiligte Schüler, zu dem Ergebnis kommen, dass die Schülerleistungen in kleinen Klassen besser sind (siehe zusammenfassend z.B. Böttcher, 2002).

Im Ergebnis führen die vorliegenden internationalen Vergleichsuntersuchungen nicht zu eindeutigen Aussagen und allenfalls zu einem schwachen (positiven) Zusammenhang zwischen Klassengröße und Schülerleistungen (vgl. u.a. Böttcher, 2002; Hanushek, 1986, 1999; Wössmann, 2002).12 Andererseits kommen Untersuchungen, die sich auf einzelne Länder beziehen, teilweise zu anderen Ergebnissen. So kommt z.B. Fertig (2003) für Deutschland zu dem Ergebnis, dass Schüler in grösseren Klassen tendenziell schwächere Leistungen haben als Schüler in kleineren Klassen.

Die geringe Evidenz für einen signifikanten Leistungssteigerungseffekt durch Verringerungen der Schüler-Lehrer-Relation könnte auch auf die Endogenität der verschiedenen Faktoren zurückzuführen sein, die statistisch kaum übergreifend bzw. vollständig erfasst werden können. Dies bedeutet, dass es keine allgemeingültige Aussage zur optimalen Klassengrösse geben kann, weil jede Klasse in Abhängigkeit von den Schülern und deren Hintergründen, Voraussetzungen und Verhalten, den Lehrern und deren Unterrichtserfahrung, -qualität und Motivation, sowie evtl. auch dem zu unterrichtenden Fach zu divergierenden Ergebnissen kommen kann (vgl. u.a. Levacic \& Vignoles, 2002; West \& Wöss- 
mann, 2003). ${ }^{13}$ Damit wäre aber die optimale Klassengröße endogen. Zwar versuchen neuere Studien, dieses Problem zu berücksichtigen, letztlich ist aber immer kritisch zu hinterfragen, ob wirklich alle relevanten Einflussvariablen tatsächlich erfasst worden sind. Insbesondere die Lehrqualität dürfte eine statistisch kaum personenübergreifend zu determinierende Variable sein.

Wössmann und West (2002) kommen allerdings auch zu dem Ergebnis, dass Klassengrösseneffekte mit dem Lehrergehalt zusammenhängen können. Danach treten Klassengrösseneffekte nur in Ländern auf, die ein relativ niedriges Gehaltsniveau haben, nicht aber in Ländern mit relativ hohen Lehrergehältern. Dieses Ergebnis führt zwangsläufig zur Frage, welchen Einfluss das Gehalt und dessen Struktur auf die Lehrqualität hat.

\section{Lehrqualität und Höhe und Struktur der Lehrergehälter}

Geht man von der ökonomischen Theorie aus, dann ist das Einkommen einerseits ein Indikator für Arbeitsproduktivität und Knappheit, die mit Angebot und Nachfrage nach einem Beruf verbunden sind und andererseits ein Anreizmechanismus im Hinblick auf die Berufsentscheidung von Personen. Wenn Personen mit höheren Qualifikationen tendenziell höhere Einkommen erhalten, dann nimmt die Attraktivität des Lehrerberufs für diese Personen mit steigendem Einkommen zu. Ist das Einkommen niedrig, dann werden sich auch eher weniger qualifizierte Personen für diesen Beruf interessieren (adverse selection). Auch wenn dies eine sehr verkürzte Betrachtung ist und Ansehen oder Beamtenstatus etc. attraktivitätsrelevant sein dürften, so implizieren die vorstehenden Ausführungen, dass das Einkommen neben anderen Faktoren ein wichtiger Indikator für die Qualität der Bewerber bzw. Lehrkräfte sein dürfte.

Wenn also Wössmann und West (2002) zu dem Ergebnis kommen, dass Klassengrößeneffekte in Ländern mit einem relativ niedrigen, nicht aber in Ländern mit einem hohen Einkommen auftreten, dann könnte dies heissen, dass bessere Lehrer auch in grösseren Klassen zurecht kommen, schlechtere aber nicht (Gundlach \& Wössmann 2003).

Tendenziell müssten dann zugleich die Schülerleistungen in internationalen Vergleichen in Ländern mit einem niedrigeren Anfangs- und/oder Endgehalt schlechter sein als in Ländern mit einem höheren Gehalt. Auf der Grundlage von TIMSS oder PISA zeigt sich diesbezüglich kein statistisch signifikanter Zusammenhang. ${ }^{14}$ Im Rahmen einer bivariaten, aggregierten Analyse zeigt sich allerdings für die Steigerungsrate des Gehalts aufgrund von Leistung oder Berufserfahrung ein signifikanter Zusammenhang $\mathrm{zu}$ den PISA-Ergebnissen (Dohmen \& Haileselassie, 2003). Dies gilt gleichermaßen für Leistungsveränderungen zwischen TIMSS und PISA. D.h. in den Ländern, in denen die Gehaltsdifferentiale geringer geworden sind, bei PISA relativ schlechter abgeschnitten haben als noch in TIMSS (z.B. Deutschland, Österreich, Schweiz), während Länder mit einer zwischenzeitlich erhöhten Prämie besser abschnitten (Frankreich, Belgien, Neuseeland). ${ }^{15}$ Es sei allerdings darauf hingewiesen, dass 
die Ergebnisse nicht widerspruchsfrei sind, so waren die Leistungen amerikanischer Schüler bei PISA schwächer als bei TIMSS, obwohl die Gehälter stärker differenziert wurden.

Da aber auch Cooper und Cohn (1997) sowie Southwick und Gill (1997) zu ähnlichen Ergebnissen kommen, also «Einheitsbezahlung» negative Effekte auf Lehrleistungen hat, während Leistungs- und/oder Erfahrungszulagen zur Verbesserung der Lehrqualität beitragen, spricht einige Evidenz für die positiven Wirkungen einer stärkerer Einkommensdifferenzierung auf die Schülerleistungen.

Abschliessend soll noch das Verhältnis von Personal- und sonstigen laufenden Ausgaben untersucht werden.

\section{Personal- versus sonstige Laufende Ausgaben}

Es erscheint plausibel anzunehmen, dass das Vorhandensein von Lehr- und Lernmaterialien einen positiven Einfluss auf die Schülerleistungen hat. In diesem Sinne würden etwa Lehrbücher oder Experimentiermöglichkeiten bessere Schülerleistungen erwarten lassen als reine Vorlesungen oder Frage-AntwortLernprozesse, d.h. Frontalunterricht. ${ }^{16}$ Demzufolge würde ein höherer Anteil an Sachausgaben die Lernergebnisse der Schüler positiv beeinflussen.

Nachdem Pritchett und Filmer (1999) diesen Zusammenhang auf der Grundlage anderer Studien für Entwicklungsländer gezeigt haben, kommen Dohmen und Haileselassie (2003) bezogen auf die Schülerleistungen bei PISA zu dem Ergebnis, dass ein höherer Anteil an Sachausgaben mit besseren Punktzahlen verbunden ist. Eine ebenso signifikante Korrelation besteht zwischen Veränderungen bei den Sachausgaben und Leistungsveränderungen zwischen TIMSS und PISA.

Diese Ergebnisse bestätigen wiederum die Schlussfolgerungen früherer Arbeiten von Fuller (1986) sowie Fuller \& Clarke (1994), ${ }^{17}$ wonach Sachausgaben relativ bedeutsamer sind als Klassengrösse oder Gehaltsausgaben. Dies würde bedeuten, dass z.B. in Deutschland, aber auch Österreich und der Schweiz eine Reallokation der Ausgaben zugunsten von mehr Sachausgaben zu besseren Schülerleistungen führen könnte.

\section{Zusammenfassung}

Die vorstehenden Ausführungen zeigen, dass keine eindeutige Korrelation zwischen der Höhe der Ausgaben und der Schülerleistung besteht. So führen höhere Ausgaben allenfalls in vergleichsweise geringem Umfang zu besseren Schülerleistungen. Dies gilt auch hinsichtlich der Verringerung der Schüler-Lehrer-Relation. Dieses Ergebnis erscheint auch insoweit plausibel als die Rahmenbedingungen bzw. Einflussfaktoren in jedem Einzelfall unterschiedlich sind, so dass anzunehmen ist, dass immer wieder adjustiert werden muss. Oder wie Worthington (2001, S. 266) es formuliert, "for a variety of reasons, not least behavioural complexities, the true relationship may never be known». 
Zwar ist zu vermuten, dass sich durch die Verbesserung der ökonometrischen Schätzverfahren zukünftig vereinzelt statistisch signifikante Zusammenhänge für bestimmte Rahmenbedingungen und Einflussfaktoren zeigen lassen, doch werden sie vermutlich immer nur für bestimmte Konstellationen Gültigkeit haben. Dies würde bedeuten, dass sich daher auch in Zukunft allgemeingültige Schlüsse vermutlich nicht werden ziehen lassen. Zurecht betont Lazear (2001) daher auch, dass die optimale Klassengrösse auch vom Schüler- und Lehrerverhalten abhängig ist, d.h. eine endogene Grösse ist.

\section{Die Organisationsstruktur von Bildungssystemen und ihr Einfluss auf Schülerleistungen}

Der aktuelle Abschnitt bezieht sich auf interne Systemfaktoren, die Einfluss auf die Schülerleistungen haben können, wie etwa die Struktur des Bildungswesens, die (weitgehend) einheitlich oder mehrgliedrig, mit zentralen oder dezentralen Prüfungen verbunden sein kann etc.

Folgt man etwa Gundlach \& Wössmann (2003) oder Wössmann (2002), dann ist mit strukturellen Massnahmen eine Leistungsverbesserung der Schüler eher zu erreichen als mit höheren Ausgaben bzw. Inputfaktoren. Konsequenterweise fordern sie daher vor allen Dingen strukturelle Reformen.

\section{Zentralexamina}

Auf der Grundlage von theoretischen Überlegungen spricht vieles für zentrale Prüfungsverfahren, die ein überregionaler und damit vergleichbarer Indikator für die Leistungsfähigkeit von Schülern sind und somit regionale bzw. lokale (Klassen)Monopole verhindern würden. Wenn die Leistungen auf zentraler Ebene abgenommen und mit allen Schülern verglichen werden, so müssen einheitliche Standards festgelegt werden, die von den Schulen und Schülern einzuhalten sind. Bessere Schulen und Schüler werden dann besser abschneiden und können dies als marktrelevanten Faktor einsetzen. D.h. es besteht für beide Seiten, Schüler und Lehrer, ein Anreiz, sich an den Standards zu orientieren. Umgekehrt erhalten schlechte Schulen und Schüler einen Makel, der sie zwingt, sich zu verbessern. ${ }^{18}$

Erfolgt die Bewertung der Schülerleistung anhand der relativen Leistungsfähigkeit in einer Klasse, dann können leistungsschwache Schüler versuchen, bessere Schüler zu beeinflussen oder den Unterricht zu stören. Daher sieht Wössmann (2002) drei zentrale Vorteile von zentralen Examina: erhöhte externe Anerkennung und Belohnung, geringerer Gruppendruck in der Klasse und verbessertes Leistungs-Monitoring von Schülern und Lehrern.

Betrachtet man die vorliegenden empirischen Ergebnisse, dann scheinen diese, anders als in den vorherigen Kapiteln, recht eindeutig darauf hinzuweisen, dass Zentralexamina einen positiven Einfluss auf die Schülerleistungen haben. 
So zeigen internationale, aber auch nationale Vergleichsuntersuchungen, dass Schüler in Bildungssystemen mit zentralisierten Prüfungsverfahren signifikant bessere Leistungen aufweisen als Schüler, deren Leistungen anhand des lokalen Leistungsdurchschnitt gemessen werden (vgl. u.a. Büchel, Jürges \& Schneider 2003, Gundlach \& Wössmann 2003; Wössmann, 2002).

\section{(Weitgehend) Einheitliches Schulsystem}

Ein normativ besetztes und diskutiertes Thema ist, ob Bildungssysteme mit oder ohne Sortierungsfunktion oder Selektion der Schüler in unterschiedlichen Leistungsebenen zu (durchschnittlich) besseren Schülerleistungen führen oder nicht. Dieses Thema wird ferner nicht nur politisch, sondern auch theoretisch kontrovers diskutiert.

So zeigt z.B. Lazear (2001) theoretisch m.E. durchaus überzeugend, dass eine Sortierung der Schüler in leistungsstärkere und leistungsschwächere unter ökonomischen Effizienzüberlegungen im Hinblick auf die akademische Leistungsfähigkeit vorteilhaft sein kann. Er geht bei seinen Überlegungen davon aus, dass leistungsstarke Schüler in grösseren Klassen unterrichtet werden können, während schwächere oder benachteiligte Schüler kleinere Klassen benötigen.

Eine entgegengesetzte Überlegung ist, dass im Falle solch homogener Klassen die Chance, dass schlechtere Schüler von den guten lernen, wesentlich geringer ist. Insofern würde ein segregierendes Schulsystem dazu führen, dass es zu pertinenten sozialen Schichtstrukturen durch Bildungs- und Einkommensunterschiede kommt, da die besseren Schüler von solchen Strukturen profitieren, während die schlechteren dadurch verlieren.

Bezogen auf US-amerikanische Schüler kommt Fertig (2002) tatsächlich zu dem Ergebnis, dass die Schülerleistungen in homogeneren Gruppen tendenziell besser sind als in heterogenen. Geht man davon aus, dass ein differenzierendes (mehrgliedriges) Schulsystem mit homogeneren Schülergruppen verbunden ist, wäre dies eine Bestätigung des bestehenden Bildungssystems u.a. in Deutschland. Dies gilt nach Fertig (2003) umso mehr, als auch bei deutschen Schülern die Leistungen in homogenen Gruppen besser sind.

Kritisch ist in diesem Kontext aber anzumerken, dass gerade Deutschland durch einen starken Zusammenhang zwischen sozioökonomischem Hintergrund und Schülerleistungen gekennzeichnet ist, so dass diese Ergebnisse mindestens unter sozialpolitischen, aber auch längerfristigen ökonomischen Überlegungen problematisch sein können. ${ }^{19}$

In verschiedenen Arbeiten wurde auch die Bedeutung der Schulautonomie diskutiert.

\section{Schulautonomie}

Ein Aspekt, der in den vergangenen Jahren auch in Deutschland und seinen Nachbarländern verstärkt Aufmerksamkeit gefunden hat, ist Schulautonomie. Wössmann (2002) sieht zwei gegenläufige Argumentationsmuster im Zu- 
sammenhang mit Schulautonomie. Einerseits wäre dezentrale Entscheidungsbefugnis vorteilhaft, da der Ressourceneinsatz in Abhängigkeit von den Bedingungen vor Ort erfolgen könne. Dies würde das Wettbewerbselement stärken und die Anpassung an die Elternvorstellungen ermöglichen, zumal auch die Lehrund Lernerfordernisse und -optionen der jeweiligen Schülerschaft berücksichtigt und adressiert werden könnten. Es dürfte für eine zentrale Administration wesentlich schwieriger, wenn nicht gar fast unmöglich sein, die konkreten Anforderungen der jeweils individuellen Situation der einzelnen Schulen sachgerecht zu berücksichtigen.

Andererseits könnte Schulautonomie aber auch dazu führen, dass sich Schulen opportunistisch verhalten, wenn Entscheidungen nicht kontrolliert und evaluiert werden bzw. Fehlverhalten nicht mit negativen Sanktionen verbunden ist. So könnten z.B. Entscheidungen über den Einsatz von Ressourcen zugunsten der Bildungsproduzenten (Lehrer) getroffen werden, während die Leistungsfähigkeit der Schüler hiervon nicht profitiert. Insgesamt wäre damit der leistungssteigernde Effekt von Schulautonomie nicht eindeutig, sondern abhängig von der konkreten Ausgestaltung. In diese Richtung weisen auch die Ergebnisse empirischer Untersuchungen.

So kommt Wössmann (2002) beispielsweise zu dem Ergebnis, dass zentralisierte Curricula und Textbuch-Genehmigung zu etwas besseren Schülerergebnissen führen. Auch Schulen, die über die Höhe des Budgets hauptsächlich selbst entscheiden konnten, wiesen durchschnittlich schwächere Schülerleistungen bei TIMSS auf als Schulen, die diese Zuständigkeit nicht hatten. Umgekehrt konnten Schulen mit Autonomie in Entscheidungen hinsichtlich Lehr- und Lernmaterialen oder Personalfragen bessere Schülerleistungen vorweisen. Dies gilt auch, wenn die Schulen über das Gehalt der Lehrer entscheiden konnten. Wössmann (2002) schliesst aus den Ergebnissen, dass die Zuständigkeit in Personalfragen von erheblicher Bedeutung für die Leistungsfähigkeit von Schulen ist. Dies gilt allerdings explizit nicht bezüglich der Höhe des Budgets oder der Lernstandards, diese sollten auf zentralerer Ebene verankert werden. ${ }^{20}$

Es spricht daher einiges dafür, Schulautonomie mit anderen Massnahmen, z.B. zentralen Examina und Festlegung von Bildungsstandards zu kombinieren und die Budgethoheit nur in sehr eingeschränktem Umfang unmittelbar an die Bildungseinrichtungen zu übertragen. Hier könnten jedoch z.B. Entscheidungen über neu einzustellende Lehrer oder die Anschaffung von Bücher oder anderen Lehr- und Lernmitteln getroffen werden. Die Budgethoheit sollte demgegenüber auf zentralere Einheiten übertragen werden, wobei zu berücksichtigen ist, dass auch diese eigene Interessen entfalten können, die zu Überbürokratisierung und einer zu geringen Informationsdichte etc. führen (Hoxby, 1999; Wössmann, 2002). 


\section{Der Einfluss von wettbewerbsorientierten Bil- dungsfinanzierungsmodellen auf Schülerleistungen}

Die Diskussion über den Beitrag der Bildungsfinanzierung und der verschiedenen ihr zugrundeliegenden Modelle ist in den vergangenen Jahren immer heftiger und in weiten Teilen ideologisch oder zumindest stark normativ geführt worden. Letzteres macht es insbesondere mit Blick auf die Einschätzung der Validität der (empirischen) Ergebnisse von Modellversuchen ausgesprochen schwierig, deren «wirkliche» Vor- und Nachteile, Erfolge und Misserfolge zu bewerten. Dies gilt vor allen Dingen mit Blick auf US-amerikanische Untersuchungen (siehe hierzu auch Levin, 2001).

Der folgende Abschnitt will versuchen, sich zwischen diesen beiden Linien zu bewegen, d.h. seine Intention ist, die Vor- und Nachteile anhand der vorliegenden Evidenz der Ergebnisse möglichst «objektiv» darzustellen und zu analysieren. Um ein Teil des Ergebnisses bereits vorwegzunehmen: Es gibt einerseits bisher keine eindeutige und unstrittige Evidenz dafür, dass markt- und wettbewerbsorientierte Finanzierungsmodelle, wie etwa Bildungsgutscheine, dem herkömmlichen (zentral-)staatlichen Finanzierungs- und Organisationsprinzip grundsätzlich und uneingeschränkt überlegen sind. Andererseits gilt aber auch das Gegenteil, dass sie diesen nicht unterlegen sind.

Bevor sich der Beitrag aber den Wirkungen unterschiedlicher Finanzierungsinstrumente widmet, erscheint es angebracht, die verschiedenen Modelle kurz in ihren Grundzügen und hinsichtlich der verfolgten Intention darzustellen. Hierbei verwenden wir den Begriff Bildungsgutschein stellvertretend für alle Formen von Finanzierungssystemen, die von einem marktwirtschaftlicheren bzw. wettbewerblicheren Ansatz ausgehen als das in Zentraleuropa vorherrschende Bildungssystem.

\section{Grundzüge von Bildungsgutscheinen und Pro-Kopf- Zuwe isungen}

Bildungsgutscheine und Pro-Kopf-Zuweisungen unterscheiden sich faktisch nur in Nuancen, die allerdings für die Wirksamkeit entscheidend sein können. ${ }^{21}$

Bei der Pro-Kopf-Zuweisung erhält die Schule die Finanzzuweisung direkt durch den Staat auf der Grundlage der gemeldeten Schülerzahlen. Das heisst, die Schule teilt dem zuständigen Ministerium die Anzahl der angemeldeten Schüler mit und erhält im Gegenzug den entsprechenden Finanzbetrag überwiesen.

Beim Bildungsgutschein erhält die Schülerin bzw. der Schüler (oder die Eltern) den Gutschein vom Staat ausgehändigt. Die Austeilung beginnt bei Beginn der Schulpflicht. Mit diesem Gutschein gehen Eltern und Schüler zu der Schule, für die sie sich entschieden haben, und übergeben ihn zusammen mit der Anmeldung. Die Schule ihrerseits leitet diesen Gutschein an das zuständige Ministerium weiter und erhält dafür im Gegenzug den entsprechenden Geldbetrag.

Der wesentliche Unterschied zwischen den beiden Instrumenten besteht so- 
mit in dem Coupon (vinkuliertes Namenspapier), den der Schüler bzw. die Schülerin im Gutscheinsystem erhält. Bei Pro-Kopf-Zuweisungen wird dieser Coupon nicht ausgegeben. Betrachtet man ausschliesslich diesen formalen Aspekt, so könnte man versucht sein, sich für die Pro-Kopf-Zuweisungen auszu-sprechen, da sie in der Administration etwas weniger aufwendig zu sein scheinen.

Andererseits stellt sich jedoch die Frage, ob der Coupon, der aktiv übergeben werden muss, einen psychologischen Vorteil dadurch bekommt, dass die Übergabe bewusst erfolgt. Hierdurch wird der Zusammenhang zwischen der Entscheidung für eine bestimmte Schule und der Finanzierung im Vergleich zu ProKopf-Zuwendungen offensichtlicher. Zugleich dürfte die verbesserte Transparenz bedeuten, dass die Entscheidung für oder gegen eine Schule in der Regel ebenfalls bewusster erfolgt, etwa dergestalt wie «Ich entscheide mich für diese Schule, weil sie meines Erachtens besser ist als andere» oder «weil ihr Fächerangebot mir am besten gefällt». Wenn der Zusammenhang transparenter und die Entscheidung bewusster getroffen wird, dann würde dies eine etwas rationalere Entscheidung auf Seiten der Beteiligten für oder gegen eine bestimmte Schule bedeuten. Dieser Zusammenhang ist bei der Pro-Kopf-Zuweisung nicht unmittelbar erkennbar, sodass ein Vorteil der Gutscheine in diesem psychologischen Effekt zu sehen ist.

Angesichts dieses Vorteils wird im Folgenden der Begriff Gutschein verwendet, da es das m.E. vorzuziehende Instrument ist. Die dargestellten Grundprinzipien gelten jedoch uneingeschränkt auch für die Pro-Kopf-Zuweisung, sodass damit keine Beschränkung auf den Gutschein verbunden ist.

In der internationalen Diskussion werden vor allen Dingen drei Erwartungen bzw. Ziele mit der Einführung von Gutscheinen verbunden:

1. sozialpolitische Ziele,

2. Leistungssteigerung durch Wettbewerb zwischen Einrichtungen,

3. mehr Wahlfreiheit.

Die Verfolgung sozialpolitischer Ziele ist eines der vorherrschenden Begründungsmuster für die Einführung von Bildungsgutscheinen. Dies bedeutet zugleich, dass die Höhe der Gutscheinwerte entweder einkommensabhängig ist oder aber die Ausgabe nur an bestimmte Einkommensgruppen erfolgt. ${ }^{22}$

So werden Bildungsgutscheine in einigen Städten und Bundesstaaten der USA nur an einkommensschwächere Familien vergeben, damit deren Kinder Zugang zu besseren und meist teureren (Privat-)Schulen erhalten können (Belfield, 2002; Rouse 1997, 1998; Witte, Thorn \& Sterr, 1995, Witte et al. 1994, Witte 1997). Auch in Kolumbien wurden Gutscheine eingeführt, um Schülern aus einkommensschwachen Familien den Schulbesuch an häufig besseren und teureren Privatschulen zu ermöglichen (Angrist et al., 2002; Calderón Z., 1996; West 1996). Chile führte ein umfassenderes Pro-Kopf-Finanzierungssytem ein (Gauri, 1998). 
Hierbei ist allerdings zu berücksichtigen, dass Privatschulen und Schulgebühren in all diesen Ländern ein wesentliches Element und daher nicht mit Deutschland und vielen anderen Ländern in Zentraleuropa vergleichbar sind. Dies dürfte bedeuten, dass in diesen Ländern Europas eine einkommensabhängige Differenzierung, die soziale Vor- und Nachteile ausgleichen würde, aus politischen Gründen wahrscheinlich ebenso auszuschliessen sind wie andere Modelle, die mit Schulgeld verbunden sind.

Denkbar erscheint demgegenüber jedoch eine Variante, die - ohne die Intention Schulgeld einzuführen - eine soziale Differenzierung vorsieht, um Benachteiligungen unterschiedlicher Art (Migration oder sprachliche Defizite, körperliche und geistige Behinderungen) auszugleichen, um eine soziale Durchmischung der Schülerschaft zu erreichen. So könnten z.B. erziehungsschwierige oder lernschwache Schüler ebenso wie viele ausländische Schüler einen Gutschein mit einem höheren Nennwert erhalten, wodurch die Schule die Möglichkeit hätte, die mit ihrer Aufnahme verbundenen höheren Betreuungs- und Ausbildungskosten abzudecken. Ansätze für eine solche Differenzierung gibt es z.B. im bayerischen Kita-Finanzierungsmodell (Krauss, 2002) ebenso wie im Gutscheinmodell im schwedischen Nacka (Ahlberg, 2002) bzw. in den Niederlanden (Patrinos 2002; Ritzen et al., 1997; de Vijlder, 2002).

Daneben verbleiben noch zwei weitere Ansätze. Entweder wird ein nach Schultypen und Schulformen differenzierter Gutschein eingeführt oder aber ein Zeitgutschein, bei dem die unterschiedlichen Kosten pro Schule über die unterschiedliche Festsetzung der Finanzzuweisung geregelt wird.

Die Zielsetzung eines solchen Modells besteht dann vor allem darin, über den Ansatz «mehr Schüler gleich mehr Geld» die Schulen zu besseren Leistungen zu motivieren. Dies ist dann zu erwarten, wenn die Schüler und ihre Eltern auf der Grundlage von qualitativen Kriterien über die zu besuchende Schule entscheiden. In diesem Fall hätten Schulen, die eine «bessere Lehrqualität» aufweisen, eine höhere Nachfrage und eine bessere Finanzausstattung als Schulen mit einer schwächeren Performance. ${ }^{23}$ Da Letztere aber auch die Lehrergehälter und die sonstigen Kosten bezahlen müssten, hätten sie einen Anreiz, ihre Leistung zu verbessern. Da gleichzeitig die «besseren» Schulen ihre Position verteidigen wollen, würden auch sie sich anstrengen, ihr Leistungsniveau zu verbessern. Auf diesem Wege käme ein leistungssteigernder Wettbewerb in Gang, der letztlich zu einem höheren Niveau des gesamten Schulsystems führen würde. Ob ein solches Szenario realistisch ist, hängt letztlich vor allen Dingen davon ab, ob Eltern und Schüler ihre Schule wirklich aufgrund von Qualitätskriterien auswählen, worauf an anderer Stelle eingegangen wird. 24

Die dritte mit der Einführung von Gutscheinen verbundene Forderung bzw. Erwartung, die Erhöhung der Wahlfreiheit, wird vor allen Dingen in den USA angeführt und hat dort einen bisweilen ideologischen Charakter. Da dies zum Teil auch für die wissenschaftliche Diskussion und Evaluation gilt, wird eine sachgerechte und unvoreingenommene Diskussion der Wirkungen von und Er- 
fahrungen mit Gutscheinmodellen erheblich erschwert, sofern man die entsprechende Grundposition der Verfasser nicht kennt.

\section{Erfahrungen mit Bildungsgutscheinen}

In diesem Abschnitt werden die im vorangegangenen Kapitel dargestellten Erwartungen einer kritischen Analyse unterzogen und Rahmenbedingungen diskutiert, die für die Wirksamkeit von Gutscheinen von Bedeutung sind bzw. sein können. 25

Die zentrale Erwartung im Hinblick auf die Einführung von Gutscheinen ist die Annahme, dass mehr Wettbewerb zu einer Leistungssteigerung im Schulsystem führt. Wie aufgrund der theoretischen Analyse zu erwarten, weisen empirische Studien einen solchen Zusammenhang nach. So ermittelt etwa Wössmann (2002) auf der Grundlage der TIMSS-Ergebnisse einen Leistungsvorteil durch Wettbewerb (mit privaten Schulen).

In einer neuen Arbeit kommt auch Hoxby (2002a) für drei School-choice Projekte in den USA (Milwaukee, Arizona und Michigan) zu dem Ergebnis, dass die Einführung bzw. Verbesserung der Schulwahlmöglichkeiten zu erheblichen Produktivitätssteigerungen und teilweise auch Leistungsverbesserungen geführt habe. Würden diese Produktivitätseffekte vernachlässigt, dann würden die positiven Wirkungen von Gutscheinen unterschätzt.

Etwas kritischer sind die Befunde von Rouse (1997, 1998) hinsichtlich der Leistungsverbesserung von Schülern, die am Milwaukee School Choice Programm teilgenommen haben. Sie kann nur leichte Verbesserungen in Mathematik, nicht jedoch bei der Lesefähigkeit feststellen. Ähnlich geringe Leistungsverbesserungen bzw. uneinheitliche Ergebnisse misst auch McEwan (2000) für Kinder aus armen Minderheitenfamilien. ${ }^{26}$

$\mathrm{Zu}$ einem vorsichtig positiven Ergebnis kommen auch Belfield und Levin (2002) in einer Auswertung von 25 Studien zu den Effekten von mehr Wettbewerb auf die akademische Leistungsfähigkeit der Schüler. «Increased competition and higher educational quality are positively correlated. [...] However, [...] the effects of competition on educational outcomes appear substantively modest» (S. 39). 27

Im Modellversuch mit dem Pro-Kopf-Finanzierungssystem der bayerischen Kindertagesstätten nehmen Eltern durchaus eine bessere Leistungsqualität wahr (Dohmen, 2002c; Krauss, 2002), wobei dies nicht anhand von Leistungsmessungen untersucht worden ist.

Im Hinblick auf mögliche sozialpolitisch relevante Konsequenzen solcher Wahlmöglichkeiten ist von Bedeutung, dass viele Eltern und Schüler weniger nach qualitativen Kriterien als vor allen Dingen nach Wohnortnähe entscheiden. So zeigen Untersuchungen in Milwaukee (Wisconsin), wo der Gutschein eingeführt wurde, um Kindern aus unteren Einkommensschichten den Besuch von Privatschulen zu ermöglichen, dass die Inanspruchnahme des Programms positiv mit dem Bildungsniveau der Eltern korreliert. d.h., dass die Eltern (insbesondere 
die Mütter) von Kindern, die für das Gutschein-Programm optiert hatten, ein höheres Bildungsniveau hatten als andere (Witte et al., 1994; Witte \& Thorn, 1996). Dies wird auch aus Grossbritannien (West \& Pennell, 1997; West \& Sparkes, 2002) und Chile (Gauri, 1998) berichtet. Bedeutsam sind in diesem Zusammenhang auch die Hinweise darauf, dass ein Teil der Eltern ihre vorhandenen Wahlmöglichkeiten nicht ausnutzen (West \& Pennell, 1997) oder viele Eltern nicht einmal über die Qualität der Schulen in ihrer näheren Umgebung informiert sind (Gauri, 1998).28

Insgesamt scheinen diese Befunde die These von Levin (1975) zu bestätigen, der davon ausging, dass die Vorteile der Wahlmöglichkeit durch Bildungsgutscheine einseitig zugunsten der höheren sozioökonomischen Schichten verteilt sind. ${ }^{29}$

Diese unterbleibende Nutzung der vorhandenen Wahlmöglichkeiten dürfte ein wesentlicher Grund dafür sein, dass im Zusammenhang mit Gutscheinen oder wettbewerblich orientierten Finanzierungsmodellen immer wieder auf Segregationswirkungen verwiesen wird. Darüber hinaus dürfte auch die Kombination mit Schul- und Studiengebühren hierbei ein wichtige Rolle spielen, wie dies z.B. für Chile berichtet wird (Lohmann, 2002). 30 Allerdings ist auch daraufhinzuweisen, dass auch rein staatlich organisierte Bildungssysteme, wie insbesondere etwa das deutsche, es nicht vermögen, dieses Phänomen ausreichend zu bekämpfen. Vielmehr ist Deutschland nach den PISA-Ergebnissen (OECD, 2001) das Land mit dem stärksten Zusammenhang zwischen dem Leistungsvermögen der Schüler und ihrem sozioökonomischen Hintergrund. Insoweit entbehrt es nicht einer gewissen Pikanterie, wenn gerade deutsche Pädagogen oder Gewerkschafter auf die sozialen Probleme von Bildungsgutscheinen oder stärker wettbewerblich verfassten Bildungssystemen verweisen.

Diese Effekte würden die Wirksamkeit von Gutscheinen ebenso beeinflussen wie etwa Peer-group-Effekte (Adnett \& Davies, 2001), bei denen die eigene Wahlentscheidung von dem Wahlverhalten von Freunden und Bekannten bzw. Personen bestimmter Statusgruppen abhängig gemacht wird.

Wichtig erscheint in diesem Kontext aber auch die Tatsache, dass die Leistungsfähigkeit von Schülern deutlich positiv mit sozioökonomischen Faktoren korreliert. Können die Schulen die Schüler ausschliesslich nach eigenem Gusto auswählen, dann dürfte dies fast zwangsläufig zu einer unerwünschten sozialen Selektion führen. Man könnte dies jedoch dadurch verhindern, dass die Schulen einem Kontrahierungszwang unterliegen, d.h. jeden Schüler annehmen müssen, der sich an dieser Schule anmelden möchte. ${ }^{31}$

Umgekehrt könnte ein nach sozialen Kriterien differenzierter Gutschein allerdings dazu genutzt werden, um Schulen einen Anreiz zu geben, Kinder mit unterschiedlichen sozioökonomischen Hintergründen oder sonstigen Benachteiligungen aufzunehmen. Der vergleichsweise geringe administrative Aufwand könnte ein Vorteil von Gutscheinen sein. 
Als weiteres Gegenargument wird behauptet, dass die Kosten eines Gutscheinsystems wesentlich höher seien als die des heutigen Systems (so etwa Mangold, Rhyn \& Oelkers, 1998, 2000; Rhyn, 2002).

Die meines Wissens einzige Kostenberechnung für ein umfassendes Bildungsgutscheinmodell im Schulbereich wurde von Levin und Driver (1997) vorgenommen. Unter Bezugnahme auf die Situation in den USA ermitteln sie um $25 \%$ höhere Kosten, wobei insbesondere die veranschlagten Transport- und Unterbringungskosten zu Buche schlagen. Diese sind nach ihrer Auffassung zu berücksichtigen, um allen Schülern die Möglichkeit zu geben, sich auch für entfernte Schulen zu entscheiden. Nur dadurch würden sie die wirkliche Schulwahlfreiheit haben.

Allerdings schränken Levin und Driver (1997) ihre Berechnung selbst insofern ein, als sie darauf hinweisen, dass es weder möglich ist, allgemein gültige Berechnungen anzustellen noch allgemein gültige Schlussfolgerungen zu ziehen, da beides von den konkreten Spezifikationen und Rahmenbedingungen des Einzelfalles abhängig ist. Es ist daher m.E. nicht zulässig, pauschal zu behaupten, dass ein Gutscheinsystem notwendigerweise teurer würde als das heutige System. Es sind jedoch ergänzende Institutionen erforderlich (Beratung, Informationen etc.), die die Funktionsfähigkeit von markt- und wettbewerbsorientierten Finanzierungssystemen erst gewährleisten. Umgekehrt ist allerdings auch zu fragen, ob das Fehlen entsprechender Institutionen im heutigen System nicht mit suboptimaler Funktionsfähigkeit verbunden ist. Dies heisst, es wären die jeweiligen vollen Kosten miteinander zu vergleichen. Dennoch ist durchaus wahrscheinlich, dass Gutscheine oder Wettbewerb nicht kostenlos zu haben sind, wie dies Mayer und Peterson (1999) behaupten.

\section{Zusammenfassung: Sind Gutscheine effizienter?}

Betrachtet man die vorliegenden Ausführungen, dann bestätigen sie die bereits einleitend formulierte Feststellung, dass Gutscheine weder eindeutig besser noch eindeutig schlechter als andere Finanzierungsinstrumente abschneiden.

Geht man von den konkreten Wirkungen von Gutscheinen bzw. vergleichbaren Finanzierungsmodellen auf die Schülerleistung aus, dann ergibt sich ein eher geringer Effizienzgewinn im Vergleich zu herkömmlichen Modellen. Wettbewerb, ob mit oder ohne Gutscheine scheint die Schülerleistungen positiv zu beeinflussen (vgl. auch Wolter, 2001). Allerdings ist darauf hinzuweisen, dass diese Auffassung auch unter Bildungsökonomen nicht unumstritten sind. So vertritt etwa Weiss (1998, 1999, 2003) wiederholt die Auffassung, dass die Evidenz für leistungssteigernde Effekte durch Wettbewerb sehr begrenzt ist (so auch Levacic \& Woods, 2000). Keineswegs ist jedenfalls der leistungssteigernde Effekt so gross, wie viele Befürworter gerne vorgeben (Hoxby, 2002a).

Nicht zu vernachlässigen sind jedoch mögliche sozialpolitische Probleme, die durch Ausgestaltung oder das unterschiedliche Wahlverhalten bzw. die Bereitschaft hierzu resultieren können. Gutscheine müssen daher die unterschiedliche 
Finanzkraft der Familien, aber auch unterschiedliche Belange der Schüler ausreichend berücksichtigen. Allerdings besteht m.E. auch kein Anlass, Gutscheine grundsätzlich und mit der Heftigkeit abzulehnen, wie dies beispielsweise Lohmann (2002) tut. Viele markt- oder wettbewerblicher ausgerichtete Bildungssysteme haben bei internationalen Leistungsvergleichen besser abgeschnitten als Länder mit staatlichen verwalteten Bildungssystemen. Information und Beratung (gerade für bildungsferne Familien) sind aber wichtige Bestandteile, die bei der Einführung berücksichtigt werden müssen (Dohmen, 2003a).

Es spricht allerdings vieles dafür, dass die alleinige Einführung von Wettbewerb - ob mit oder ohne Gutscheine - weniger effektiv ist, wenn dies nicht mit anderen Elementen kombiniert wird.

\section{Zusammenfassung und Schlussfolgerung}

In den vorangegangenen Abschnitten wurden drei Ansätze hinsichtlich ihrer Bedeutung für eine Effizienzverbesserung des Bildungssystems betrachtet und diskutiert.

Hierbei ist die Bildungsproduktionsfunktion eine Mischung zwischen einem eher mikroökonomischen und einem systemischen Ansatz, die den Zusammenhang zwischen den Inputfaktoren und den Outputgrössen misst. Eine wichtige Frage hierbei ist, ob zusätzlich finanzielle Ressourcen mit besseren Schülerleistungen korrelieren. Die bisherigen empirischen Ergebnisse deuten allenfalls auf einen schwachen Zusammenhang hin. Dies gilt im Wesentlichen auch für eine (generelle) Verringerung der Schüler-Lehrer-Relation. Vielmehr scheint einiges dafür zu sprechen, dass hier eine gezielte Intervention unter bestimmten Voraussetzungen (z.B. sprachliche, körperliche, geistige oder soziale Benachteiligungen) sinnvoller ist. Von begrenzter Aussagekraft sind bisher noch Ansätze, die die Ressourcenallokation z.B. auf Gehälter und Sachausgaben oder die Gehaltsdifferenzierung aufgrund von Leistung oder Berufserfahrung betrachten. Diese bisherigen, vorläufigen Ergebnisse weisen aber auf eine interessante, d.h. statistisch signifikante Beziehung hin.

Von deutlich stärkerer Signifikanz sind Untersuchungen zur Wirksamkeit von strukturellen Rahmenbedingungen. So führen zentrale Prüfungsverfahren offenbar zu einer deutlichen Leistungssteigerung bei den Schülerinnen und Schülern. Auch Prozess- und Personalverantwortung auf schulischer Ebene scheinen die Leistungsfähigkeit zu verbessern, während Budgetkompetenz und kollektive Interessenvertretung einen negativen Einfluss zu haben scheinen. Uneindeutig sind noch die Befunde hinsichtlich längerer gemeinsamer Bildungswege, d.h. Differenzierung der Schülerschaft in unterschiedliche (vertikale) Bildungseinrichtungen. Unter Einbeziehung auch der IGLU-Ergebnisse (Bos u.a., 2003) scheint allerdings einiges für spätere Differenzierung bei verbesserter Förderung schwächerer und/oder benachteiligter Schüler zu sprechen. 
Betrachtet man die Effekte, die von einer Neugestaltung der Finanzierung in Richtung auf Gutscheine oder Pro-Kopf-Zuweisungen ausgehen, d.h. mit (bedingtem) Wettbewerb zwischen Bildungseinrichtungen verbunden sind, dann scheinen auch sie mit leistungsstimulierenden Wirkungen verbunden zu sein. Allerdings ist die Korrelation mit den Schülerleistungen weniger stark ausgeprägt als theoretisch zu erwarten wäre.

Aus einer sinnvollen gleichzeitigen Modifikation von strukturellen und finanziellen Rahmenbedingungen könnte somit ein stärkerer Impuls für eine Effizienzsteigerung von Bildungssystemen ausgehen. Wenn die Höhe der Ressourcen, die einer Schule zur Verfügung stehen, zumindest teilweise von der Anzahl der Schüler abhängt, und deren Leistungen zentral überprüft werden, dann haben Eltern und Schüler, aber auch Lehrer und Administratoren einerseits eine Kontrolle und andererseits auch Informationen über die Schülerleistungen an dieser Schule. Es erscheint höchst unwahrscheinlich, dass Eltern und Schüler, aber auch Lehrer hierauf kollektiv nicht regieren werden. ${ }^{32}$ Es werden auf allen Ebenen, einschliesslich der Lehrer, Diskussionen einsetzen, an welche Schule man gehen soll bzw. wie man die Lehrqualität und damit die Schülerleistungen verbessern kann.

Hierdurch würden m.E. die richtigen Anreizmechanismen gesetzt, um die in Kapitel 2 dargestellten Interessengegensätze produktiv und leistungssteigernd zu steuern.

Durch geeignete Massnahmen sind soziale Segregationswirkungen ebenso zu verhindern wie Schulschliessungen durch unzureichende (demografisch oder lokal bedingte) Nachfrage. Wenn Schulen aus qualitativen Gründen schliessen oder wirklich schlechte und «beratungsresistente» Lehrer die Schule verlassen müssen oder andere Aufgaben zugewiesen bekommen, dann ist dies im Einzelfall sicherlich unerfreulich; es stellt sich umgekehrt aber zugleich die Frage, ob es hinnehmbar ist, dass die Zukunft nachwachsender Generationen durch inkompetente oder unmotivierte Lehrer beeinträchtigt oder gar verspielt wird.

\section{Anmerkungen}

1 Im ökonomischen Sinne bedeutet Effizienz, dass ein bestimmtes Produkt zu den geringsten Kosten hergestellt wird. Umgekehrt kann auch von einer bestimmten Inputmenge, z.B. Geld, ausgegangen werden, mit der dann eine möglichst grosse Menge an Produkten zu erstellen ist. Bezogen auf das Bildungssystem wäre dies also beispielsweise die «Produktion» einer möglichst grossen Zahl an (erfolgreichen) Absolventen während eines Schuljahres. Absolventen meint damit nicht nur erfolgreiche Schulabgänger, sondern Übertritte in die nächsthöhere Jahrgangsstufe. Geht man von PISA aus, dann könnte man dies auch in eine möglichst grosse Anzahl von Schülerinnen und Schülern mit einer möglichst hohen Punktzahl transformieren. Dies ist der übliche Ansatz von Analysen zu Bildungsproduktionsfunktionen (Hanushek, 1995).

2 Der hier skizzierte Verhaltensansatz ist relativ einfach und wird in den folgenden Abschnitten teilweise verfeinert bzw. modifiziert, um auch andere Faktoren zu berücksichtigen. Hierzu zählen bei Schülern etwa kurzfristige Freizeitmaximierung oder andere Störfaktoren. 
3 Um Missverständnissen vorzubeugen: hier wird nicht unterstellt, dass alle Lehrer diesem Prinzip folgen und schlechten und unmotivierten Unterricht erbringen. Es ist aber anzunehmen, dass das Fehlen externer Anreizmechanismen dazu führt, dass die Einsatzbereitschaft geringer ist als bei deren Existenz. Ein vergleichbarer Einsatz ist lediglich bei einer sehr hohen intrinsischen Motivation zu erwarten.

4 Einschränkend ist allerdings darauf hinzuweisen, dass die wirtschaftspolitischen Interessengruppen auch andere Forderungen erheben werden als nur bildungspolitische. Dies wird tendenziell ihr «natürliches» Interesse an bildungspolitischen Themen begrenzen.

5 Die Qualität der Schüler ist allerdings dann kein externer Faktor, wenn eine Bildungseinrichtung sich ihre Schüler selbst aussuchen kann.

6 Die Anzahl der Unterrichtsstunden (vor der Klasse) ist nicht zu verwechseln mit der eigentlichen Arbeitszeit, die auch Vor- und Nachbereitung, Beratungs- und Konferenzzeiten sowie sonstige Tätigkeiten umfasst.

7 Die Instruktionszeit ist die Anzahl der Unterrichtsstunden für die Schüler, z.B. 30 oder 35 Stunden pro Woche, während die Unterrichts- oder Kontaktzeit für die Lehrer die Zeit ist, die diese vor einer Klasse stehen, also etwa 24 Stunden pro Woche.

$8 \mathrm{Zu}$ einem zusammenfassenden Überblick über frühere Studien siehe u.a. Wössmann (2002).

9 Auf aggregierter Länderebene ergibt sich für Deutschland eine positive Korrelation zwischen Bildungsausgaben und Schülerleistungen im Rahmen von PISA-E, d.h. der deutschen Ergänzungsstudie, nur, wenn die Stadtstaaten ausgeschlossen werden. Es ist allerdings darauf hinzuweisen, dass die Fallzahlen insgesamt sehr gering sind und somit hieraus keine weitergehenden Schlüsse gezogen werden können.

$10 \mathrm{Zu}$ einem vergleichbaren Schluss kommt auch Iacovou (2002) für die ersten Bildungsjahre in Grossbritannien, wobei die Gruppengrösse teilweise mit über 30 Kindern relativ gross im Vergleich zu anderen Studien ist. Sie kommt damit zu anderen Ergebnissen als vorhergehende Studien (siehe hierzu Iacovou, 2002).

11 In einer aktuellen Untersuchung haben Wössmann und West (2002) versucht, diese Probleme durch ökonometrische Verfahren (fixed effects, instrumental variables) zu umgehen.

12 Dieser Auffassung wird zwar teilweise widersprochen (Hedges, Lain \& Greenwald, 1994; Krueger, 1999), doch scheinen hierbei vor allen Dingen unterschiedliche Bewertungsmassstäbe, nicht aber wirklich unterschiedliche Ergebnisse massgeblich zu sein.

13 Darüber hinaus hat auch die Wahl des Wohnortes Einfluss auf die Schulwahl und wird somit zu einer endogenen Variable, die Auswirkungen auf die Produktionsfunktion einer Schule bzw. die Schülerleistungen haben kann (Levacic \& Vignoles, 2002).

14 Ein statistisch signifikanter Zusammenhang zeigt sich allerdings zwischen dem Anfangsgehalt und der Kontaktzeit der Lehrer, d.h. ein geringes Anfangsgehalt ist dann ein Indikator für eine geringe Kontaktzeit (Dohmen \& Haileselassie, 2003).

15 Hierbei ist allerdings zu berücksichtigen, dass die teilnehmenden Länder in beiden Studien nicht identisch sind. Da aber der Länderdurchschnitt massgeblich ist für das relative Abschneiden der Länder, können die Ergebnisse nicht unmittelbar miteinander verglichen werden. Insoweit sind die Ergebnisse mit Einschränkungen zu versehen.

16 Unabhängig von diesen Überlegungen zeigen Brown und Belfield (2002), dass Vorlesungen die kosten-effektivste Unterrichtsform sein können. Andere Unterrichtsformen mögen zwar effektiver im Hinblick auf bessere Lernergebnisse sein, allerdings wären sie auch mit (deutlich) höheren Kosten verbunden.

17 Zitiert nach Pritchett \& Filmer (1999).

18 Für einen solchen Anreiz sind allerdings verschiedene Voraussetzungen notwendig. 1. müssen sich die Nachfrager bei der Schulwahlentscheidung an Qualitätskriterien orientieren, 2. muss die Qualität mittelbar oder unmittelbar eine Rückwirkung auf die Schule haben, z.B. 
über deren Budget oder andere Sanktionen. Sind diese Voraussetzungen nicht erfüllt, ist eine Orientierung an Standards nicht unbedingt zu erwarten. Eine intrinsische Motivation der Lehrkräfte kann zu guten Lehrleistungen führen, muss aber nicht. D.h. ohne externe Anreiz- oder Sanktionsmechanismen könnten sich schlechte Schulen einer Leistungsverbesserung verweigern. Entscheiden Eltern und Schüler nicht anhand der Qualität, dann würde diese Schule jedoch keine oder nur geringe Nachfrageveränderungen erfahren.

Empirische Untersuchungen zeigen, dass rationales, d.h. qualitätsorientiertes Wahlverhalten nicht unbedingt erwartet werden kann. Vielmehr scheint ein positive Korrelation zum Bildungsniveau der Eltern zu bestehen und zwar selbst dann, wenn es sich um einkommensschwache Familien handelt (Gauri, 1998; West \& Pennell, 1997; West \& Sparkes, 2002; Witte et al., 1994; Witte \& Thorn, 1996).

19 Nach aktuellen Arbeitsmarktprognosen wird Deutschland in der Mitte der nächsten Dekade seinen Bedarf an hoch- und höherqualifizierten Arbeitskräften nicht decken können, während es bei niedrigqualifizierten Positionen zu einem Überangebot kommen wird. Aufgrund des starken Zusammenhangs zwischen sozioökonomischen Hintergrund und Schulleistungen und Studierverhalten bedeutet dies, dass vor allen Dingen das Bildungsniveau der bildungsfernen Schichten angehoben werden muss, um den Bedarf des Arbeitsmarktes decken zu können. Unterbleibt dieses aufgrund unzureichender Fördermassnahmen, dann wird Deutschland mit erheblichen wirtschaftspolitischen Problemen zu kämpfen haben, schliesslich zeigen neuere Studien, dass das Bildungsniveau einer Gesellschaft von zentraler Bedeutung für die wirtschaftliche Entwicklung ist (Gundlach \& Wössmann 2003; Wössmann, 2002).

20 In einem weiteren Schritt untersucht Wössmann (2002) auch den unmittelbaren Lehrereinfluss, der in die gleiche Richtung weist wie die vorhin beschriebenen Ergebnisse. Dies gilt allerdings nicht, wenn Lehrer kollektiv oder durch Gewerkschaften auf das Curriculum Einfluss nehmen. In diesem Fall sind die Ergebnisse deutlich schlechter (-32,3 Punkte in Mathematik und -18,4 Punkte in den Naturwissenschaften).

21 Siehe hierzu und im Folgenden ausführlicher Dohmen (2002a, 2002b und 2002c) sowie die verschiedenen Beiträge in Dohmen \& Cleuvers (Hrsg.), (2002) für die unterschiedlichen Bildungsbereiche sowie übergreifend Wolter (2001).

22 In diesem Abschnitt werden nur die Erwartungen dargestellt, die mit der Einführung von Bildungsgutscheinen und vergleichbaren Instrumenten verbunden sind. Auf Erfahrungen wird im folgenden Abschnitt eingegangen.

23 Johnes (1993, S. 121) formuliert dazu: «Schools which are better (as perceived by the market) will experience a rise in demand for their services and will, in response, increase their provision. Schools which are worse (again, as perceived by the market) will suffer a fall in demand and will therefore cut back their provision.»

24 In vielen Arbeiten wird Wettbewerb mit der Existenz privater Einrichtungen gleichgesetzt bzw. dadurch gemessen. Dies ist jedoch nicht unbedingt notwendig, wenn auch evtl. für die bessere Zielerreichung hilfreich. Wettbewerb kann grundsätzlich auch zwischen öffentlichen Einrichtungen erreicht werden, indem das Budget der Einrichtungen von Nachfrageindikatoren abhängig ist.

25 Siehe hierzu und im Folgenden ausführlicher Dohmen (2003b).

26 In Milwaukee sind seit 1998 15\% der Schüler in das Gutscheinprogramm einbezogen, während es vorher $1,5 \%$ waren. Theoretisch ist durch eine solche Ausweitung zu erwarten, dass der wettbewerbsbedingte Anreiz zur Leistungsverbesserung grösser geworden ist. Dies könnte die Ergebnisveränderungen zwischen Rouse $(1997,1998)$ und Hoxby (2002a) zumindest teilweise erklären. Die Konsequenz wäre, dass Gutscheinmodelle in grossem Umfang eingeführt werden müssten, um klare Leistungsverbesserungen zu erreichen.

27 Interessant ist die Schlussfolgerung allerdings mit Blick auf frühere Arbeiten von Belfield, in denen er die vergleichsweise geringen Leistungseffekte etwas skeptischer bewertet hat 
(siehe etwa Belfield, 2002). Dies könnte darauf hindeuten, dass sich die statistische Evidenz für Leistungsverbesserungen durch Gutscheine verbessert, ohne dass dies überbewertet werden soll.

Allerdings sei darauf hingewiesen, dass sich die Ergebnisse durch vergleichsweise geringe Modifikationen der zugrundeliegenden Annahmen bzw. Betrachtungen verändern können. So berichten verschiedene Studien z.B. das insbesondere Schüler aus MinderheitenFamilien bessere Leistungen vorweisen, wenn sie Gutscheine zur Verfügung haben (z.B. Ladd, 1999). Hierbei wir häufig auf die Nationalität der Mutter abgehoben. In einer neuen Untersuchung kommen Krueger und Zhu (2003) für das New York City School Voucher Experiment zu dem Ergebnis, dass sich diese positiven Befunde deutlich relativieren, wenn stattdessen auf die Nationalität des Vaters abgehoben wird. Dieses Ergebnis bestätigt die Aussage von Belfield und Levin (2002), dass die statistische Signifikanz durch leichte Veränderungen der Annahmen bzw. Betrachtungsebene beeinflusst werden kann und somit nicht als uneingeschränkt gültig anzusehen ist.

28 Mit Blick auf die Abschaffung des Vouchers in Grossbritannien sei angemerkt, dass er durch einen Quasi-Voucher ersetzt wurde, der wesentliche Elemente des ursprünglichen Modells übernommen hat, allerdings etwas stärker auf Kontrolle und Evaluation setzt (West \& Sparkes, 2002).

29 Allerdings sehen Epple/Romano (2002) die Möglichkeit, entsprechende Effekte durch eine gezielte Ausgestaltung zu verhindern bzw. zumindest zu minimieren.

30 Bezüglich Chile wird aber mehrfach von allenfalls geringen positiven Effekten auf die Leistungsfähigkeit der Schüler bzw. insbesondere deren soziale Selektion berichtet (Carnoy \& McEwan, 2001; Hsieh \& Urquiola, 2002; McEwan, 2001). Es ist daher davon auszugehen, dass das Voucher in Chile zu problematischen Ergebnissen geführt haben, was auf die konkrete Ausgestaltung im chilenischen Kontext zurückzuführen sein könnte.

31 Sollte diese Schule keine freien Kapazitäten mehr haben, dann muss durch ein im Vorhinein festzulegendes Verfahren sichergestellt werden, dass eine Auswahl nach sozialen Kriterien unterbleibt. So könnte etwa die Besetzung nach Einkommen und sozialen Gruppen getrennt stattfinden, d.h. die aufgenommenen Schüler müssen dann der Zusammensetzung aller Anmeldungen entsprechen. In Kolumbien oder auch im Modellversuch in Alum Rock (USA) erfolgte die Vergabe bei begrenzten Kapazitäten ebenso durch ein Losverfahren wie in Milwaukee (Gendt, 1980; Rouse, 1997).

32 So zeigen Hanushek und Rivkin (2002), Hoxby (2002b) und Rapp (2000) einen positiven Einfluss von Wettbewerb auf Lehrleistung und Lehrqualität. 


\section{Literatur}

Adnett, N. \& Davies, P. (2001). Schooling Reform in England: From Quasi-Markets to Competition. European Conference of Educational Research. Paper. September 5-8. Lille.

Ahlberg, K. (2002). The Nacka Voucher Scheme. In D. Dohmen \& B.A. Cleuvers (Hrsg.), Nachfrageorientierte Bildungsfinanzierung. Neue Trends für Kindertagesstätte, Schule und Hochschule (S. 155-161). Bielefeld: W. Bertelsmann.

Angrist, J., Bettinger, E., Bloom, E., King, E. \& Kremer, M. (2002). Vouchers for Private Schooling in Colombia: Evidence from a Randomized Natural Experience. American Economic Review, 92, (5), 1535-1559.

Belfield, C.R. (2002). International Experience with Demanded Financing: Education Vouchers in the USA, Great Britain and Chile. In D. Dohmen \& B.A. Cleuvers (Hrsg.), $\mathrm{Na}-$ chfrageorientierte Bildungsfinanzierung. Neue Trends für Kindertagesstätte, Schule und Hochschule (S. 162-181). Bielefeld: W. Bertelsmann.

Belfield, C.R. \& Levin, H.M. (2002). The Effects of Competition on Educational Outcomes: A Review of US Evidence. NCSPE Working Paper. New York: NCSPE.

Bos, W., Lankes, E.-M., Prenzel, M., Schwippert, K., Walther, G. \& Valtin R. (2003). Erste Ergebnisse aus IGLU. Schülerleistungen am Ende der vierten Jahrgangsstufe im internationalen Vergleich. Münster u.a.: Waxmann.

Brown, C., Belfield, C.R. (2002). How cost-effective are Lectures? A Review of Experimental Literature. In H.M. Levin \& P.J. McEwan (Eds.), Cost-effectiveness and Educational Policy. Yearbook of the American Education Finance Association (S. 139-156). Larchmont: Eye on Education.

Böttcher, W. (2002). Kann eine ökonomische Schule auch eine pädagogische sein? Schulentwicklung zwischen Neuer Steuerung, Organisation, Leistungsevaluation und Bildung. Weinheim: Juventa.

Büchel, F., Jürges, H. \& Schneider K. (2003). Leistungs- und Signaleffekte zentraler Abschlussprüfungen - Eine TIMSS-Auswertung bei deutschen Haupt-und Realschülern. Vortrag beim Bildungsökonomischen Ausschuss des Vereins für Sozialpolitik. Zürich, Berlin, Dortmund \& Mannheim (mimeo(.

Calderón Z.A. (1996). Voucher Program for Secondary Schools: The Colombian Experience. World Bank Human Capital Development and Operations Policy Working Papers. Washington (http)//wbln0018.worldbank.org/(.

Carnoy, M. (1995a). Political Economy of Educational Production. In M. Carnoy (Ed.), International Encyclopedia of Economics of Education (2nd ed.) (S. 291-297). Oxford: Pergamon.

Carnoy, M. (1995b). Joint Production of Education. In M. Carnoy, (Hrsg.), International Encyclopedia of Economics of Education (2nd ed.) (S. 297-303). Oxford: Pergamon.

Carnoy, M. \& McEwan, P.J. (2001). Privatization Through Vouchers in Developing Countries: The Cases of Chile and Colombia. In H.M. Levin (Ed.), Privatizing Education (S. 151-177). Cambridge: Westview Press.

Coleman, J.S., Campbell, E.Q., Hobson, C.J., McPartland, J., Mood, A.M., Weinfield, F.D. \& York, R.L. (1966). Equality of Educational Opportunity. Washington, D.C.: US Department of Health Education and Welfare.

Cooper, S.T. \& Cohn, E. (1997). Estimation of a Frontier Production Function for the South Carolina Educational Process. Economics of Education Review, 16, (3), 313-327.

Dohmen, D. (2002a). Bildungsgutscheine und Pro-Kopf-Zuweisungen - Ansätze zur Finanzierung von Schulen? In D. Dohmen \& B.A. Cleuvers (Hrsg.), Nachfrageorientierte Bildungsfinanzierung. Neue Trends für Kindertagesstätte, Schule und Hochschule (S. 122-133). Bielefeld: W. Bertelsmann.

Dohmen, D. (2002b). Hochschulfinanzierung durch Bildungsgutscheine und Pro-Kopf-Zuweisungen? In D. Dohmen, \& B.A. Cleuvers (Hrsg.), Nachfrageorientierte Bildungsfinan- 
zierung. Neue Trends für Kindertagesstätte, Schule und Hochschule (S. 200-221). Bielefeld: W. Bertelsmann.

Dohmen, D. (2002c). Aspekte der Einführung eines neuen Modells zur Finanzierung der Kinderbetreuung. Kurzgutachten im Auftrag der F.D.P.-Fraktion im Landtag Nordrhein-Westfalen. Köln (unveröffentlicht(.

Dohmen, D. (2003a). The Youth Corps Voucher in Sri Lanka. Gutachten im Auftrag der International Labour Organisation für das Ministry of Policy Development and Implementation Sri Lanka. Köln (unveröffentlicht(.

Dohmen, D. (2003b). Hochschulfinanzierung durch Bildungsgutscheine. Bielefeld: W. Bertelsmann.

Dohmen, D. \& Cleuvers, B.A. (2002). Nachfrageorientierte Bildungsfinanzierung. Neue Trends für Kindertagesstätte, Schule und Hochschule . Bielefeld: W. Bertelsmann.

Dohmen, D. \& Haileselassie, A. (2003). Die Veränderung der bildungspolitischen Position Deutschlands im internationalen Vergleich. Unveröffentlicht.

Epple, D. \& Romano, R. (2002). Educational Vouchers and Cream Skimming. National Bureau of Economic Research. Working paper 9354. Cambridge, Mass.: NBER.

Fertig, M. (2002). Educational Production, Endogenous Peer Group Formation and Class Composition - Evidence From the PISA 2000 Study. Essen (www.rwi-essen.de(.

Fertig, M. (2003). Who's to Blame? The Determinants of German Students' Achievement in the PISA 2000 Study. Essen (www.rwi-essen.de).

Figlio, D.N. (1999). Functional form and the estimated effects of school resources. Economics of Education Review, 18, 241-252.

Fuller, B. (1986). Raising school quality in developing countries: What investments boost learning? World Bank Discussion Paper, 76. Washington D.C.: (The World Bank(.

Fuller, B. \& Clarke, P. (1994). Raising school effects while ignoring culture? Local conditions and the influence of classroom tools, rules and pedagogy. Review of Educational Research, 64, (1), 119-157.

Gauri, V. (1998). School Choice in Chile: Two Decades of Educational Reform. Pittsburgh: University of Pittsburgh Press.

Gendt, M.C.E. van (1980). The Voucher Concept and the Publicness of Basic Education. Meppel: Krips Repro.

Glass, V. \& Smith, M.L. (1979). Meta-analysis of Research on Class Size and Achievement. Educational Evaluation and Policy Analysis, 1, (1), 2-16.

Gundlach, E. \& Wössmann, L. (2003). Bildungsressourcen, Bildungsinstitutionen und Bildungsqualität: Makroökonomische Relevanz und mikroökonomische Evidenz. Bildungsökonomischer Ausschuss des Vereins für Sozialpolitik. Vortrag. 29.3. Zürich, Kiel (mimeo(.

Hanushek, E.A. (1986). The economics of schooling: Production and efficiency in public schools. Journal of Economic Literature, 24, 1141-1177.

Hanushek, E.A. (1995). Education Production Functions. In M. Carnoy (Ed.), International Encyclopedia of Economics of Education (2nd ed.) (S. 277-282). Oxford: Pergamon.

Hanushek, E.A. (1999). The evidence on class size. In S.E. Mayer \& P.E. Peterson (Eds.), Earning and Learning. How Schools Matter (S. 131-168). Washington, D.C.: Brookings Institution Press In. C. Hoxby (Ed.).

Hanushek, E.A. \& Rivkin, S.G. (2003). Does Public School Competition Affect Teacher Quality?, In C. Hoxby (Ed.), The Economics of School Choice, Chicago: The University of Chicago Press.

Hedges, L.V., Laine, R.D. \& Greenwald, R. (1994). Does Money Matter? A Meta-Analysis of Studies of the Effects of Differential School Inputs on Student Outcomes. Educational Researcher, 23, (3), 5-14.

Hoxby, C.M. (1999). The Productivity of Schools and Other Local Public Goods Producers. Journal of Public Economics, 74, (1), 1-30. 
Hoxby, C.M. (2002a). Government Intervention in the Provision and Financing of Education. Meeting of Representatives of National Economic Research Organisations. Paper. July first. OECD. Paris (mimeo(.

Hoxby, C.M. (2002b). Would School Choice Change the Teaching Profession? The Journal of Human Resources, 37, (4), 846-891.

Hsieh, C.-T. \& Urquiola, M. (2002). When schools compete, how do they compete? Education Occasional Paper No. 43. National Center for the Study of Privatization (www.ncspe.org(.

Iacovou, M. (2002). Class Size in the Early Years: Is Smaller Really Better? Education Economics, 10, (3), 261-290.

Ingenkamp, K., Petillon, H. \& Weiss M. (1985). Klassengrösse: Je kleiner desto besser? Weinheim \& Basel: Beltz.

Johnes, G. (1993). The Economics of Education. Houndsmills: Macmillan.

Krauss, G. (2002). Erprobung eines neuen Fördermodells für Kindertageseinrichtungen in Bayern. In D. Dohmen \& B.A. Cleuvers (Hrsg.), Nachfrageorientierte Bildungsfinanzierung. Neue Trends für Kindertagesstätte, Schule und Hochschule (S. 63-83). Bielefeld: W. Bertelsmann.

Krueger, A.B. (1999). An Economist's View of Class Size Research. Princeton (mimeo(.

Krueger, B. \& Zhu P. (2003). Another Look at the New York City School Voucher Experiment . NCSPE-Working paper, 65 (www.ncspe.org.(.

Ladd, H.F. (1999). The Dallas school accountability and incentive program: an evaluation of ist impacts on student outcomes. Economics of Education Review, 18, 1-16.

Lazear, E.P. (2001). Educational Production. Quarterly Journal of Economics, CXVI, (3), 777803.

Leva_i_, R. \& Woods, P.A. (2000). Quasi-markets and school performance: evidence from a study of English secondary schools. In M. Weiss \& H. Weishaupt (Hrsg.), Bildungsökonomie und Neue Steuerung (S. 53-95). Frankfurt/Main u.a.: Peter Lang.

Leva_i_, R. \& Vignoles, A. (2002). Researching the Links between School Resources and Student Outcomes in the UK: A Review of Issues and Evidence. Education Economics, 10, (3), 313-331.

Levin, H.M. (1975). Educational Vouchers and Educational Equality. In M. Carnoy (Ed.), Schooling in a Corporate Society (2nd ed.) New York.

Levin, H.M. \& Driver, C.E. (1997). Costs of an Educational Voucher System. Education Economics, 5, (3), 265-283.

Levin, H.M. (2001), Studying Privatization in Ecucation. In H.M. Levin (Ed.), Privatizing Education (S. 3-19) Cambridge: Westview Press.

Lohmann, I. (2002). After Neoliberalism. Können nationalstaatliche Bildungssysteme den ,freien Markt' überleben? In I. Lohmann \& R. Rilling (Hrsg.), Die verkaufte Bildung (S. 89-107). Opladen: Leske + Budrich.

Mangold, M., Oelkers, J. \& Rhyn, H. (1998). Die Finanzierung des Bildungswesens durch Bildungsgutscheine - Modelle und Erfahrungen. Studie im Auftrag der Erziehungsdirektoren des Kantons Bern. Bern: Erziehungsdirektion.

Mangold, M., Oelkers, J. \& Rhyn, H. (2000). Bildungsfinanzierung durch Bildungsgutscheine. Modelle und Erfahrungen. Zeitschrift für Pädagogik, 1, 39-60.

Mayer, S.E. \& Peterson P.E. (1999). The Costs and Benefits of School Reform. In S.E. Mayer \& P.E. Peterson (Eds.), Earning \& Learning. How Schools Matter (S. 341-354). Washington D.C.: Brookings Institution Press, Russell Sage Foundation.

McEwan, P. (2000). The potential impact of large-scale voucher programs. National Centre for the Study of Privatization in Education. Working paper. New York (www.ncspe.org.(.

McEwan, P. (2001). The Effectiveness of Public, Catholic, and Non-Religous Private Schools in Chile's Voucher System. Education Economics, 9, (2), 103-128.

Odden, A. (1990). Class Size and Student Achievement: Reseach-based Policy Alternatives. 
Educational Evaluation and Policy Analysis, 12, (2), 213-227.

OECD (2001). Lernen für das Leben. Erste Ergebnisse von PISA 2000. Paris: OECD.

OECD (2002). Reading for Change. Performance and Engagement across Countries - Results from PISA Paris: OECD.

Patrinos, H.A. (2002). Private Education Provision and Public Finance. The Netherlands as a Possible Model. National Center for the Study of Privatization in Education, Occasional Paper 59 (www.ncspe.org.(.

Pritchett, L. \& Filmer, D. (1999). What education production function really show: a positive theory of education expenditures. Economics of Education Review, 18, 223-239.

Rapp, G.C. (2000). Agency and Choice in Education: does school choice enhance the work effort of teachers? Education Economics, 8, (1), 37-63.

Rhyn, H. (2002). Bildungsgutscheine zur Reparation maroder Schulsysteme? In D. Dohmen \& B.A. Cleuvers (Hrsg.), Nachfrageorientierte Bildungsfinanzierung. Neue Trends für Kindertagesstätte, Schule und Hochschule (S. 182-185). Schriften zur Bildungs- und Sozialökonomie, 1. Bielefeld: W. Bertelsmann.

Ritzen, J.M.M., Dommelen, J. van \& Vijlder, F.J. de (1997). School Finance and School Choice in the Netherlands. Economics of Education Review, 16, (3), 329-335.

Rouse, C.E. (1997). Private School Vouchers and Student Achievement: An Evaluation of the Milwaukee Parental Choice Program. National Bureau of Economic Research, Working Paper 5964. Cambridge, Mass.: NBER.

Rouse, C.E. (1998). Private School Vouchers and Student Achievement: An Evaluation of the Milwaukee Parental Choice Program. Quarterly Journal of Economics, 113, 553-602.

Southwick, L.Jr. \& Gill, I.S. (1997). Unified Salary Schedule and Student SAT Scores: Adverse Effects of Adverse Selection in the Market for Secondary School Teachers. Economics of Education Review, 16, (2), 143-153.

Vijlder, F.J. de (2002). Choice and Financing of Schools in the Netherlands: The art of maintaining an open system responsive to its changing environment. In D. Dohmen \& B.A. Cleuvers (Hrsg.), Nachfrageorientierte Bildungsfinanzierung. Neue Trends für Kindertagesstätte, Schule und Hochschule (S. 134-154). Schriften zur Bildungs- und Sozialökonomie, 1. Bielefeld: W. Bertelsmann.

Weiss, M. (1998). Schulautonomie im Licht mikroökonomischer Bildungsforschung. In R.K. von Weizsäcker (Hrsg.), Deregulierung und Finanzierung des Bildungswesens (S. 15-47). Schriften des Vereins für Sozialpolitik, 262. Berlin: Duncker \& Humblot.

Weiss, M. (1999). Mehr Effizienz im Schulbereich durch dezentrale Ressourcenverantwortung und Wettbewerbssteuerung? Recht der Jugend und des Bildungswesens, 4, 413-423.

Weiss, M. (2003). Kann das Schulwesen durch Wettbewerb genesen? In H. Döbert, B. von Kopp, R. Martini \& M. Weiss (Hrsg.), Bildung vor neuen Herausforderungen (S. 111-119). Neuwied: Luchterhand.

West, A. \& Pennell, H. (1997). Educational Reform and School Choice in England and Wales. Education Economics, 5, (3), 285-305.

West, A. \& Sparkes, J. (2002). Examining the impact of nursery education vouchers and quasi-vouchers in England. In D. Dohmen \& B.A. Cleuvers (Hrsg.), Nachfrageorientierte Bildungsfinanzierung. Neue Trends für Kindertagesstätte, Schule und Hochschule (S. 84-99). Schriften zur Bildungs- und Sozialökonomie, 1. Bielefeld: W. Bertelsmann.

West, E.G. (1996). Education vouchers in practice and principle: a world survey (http://www.wordbank.org/html/extdr/hnp/hddflash/workp/wp_00064.html(.

West, M.R. \& Wössmann, L. (2003). Which School System Sort Weaker Students into Smaller Classes? International Evidence. Kiel Working Paper 1145. Kiel: Institut für Weltwirtschaft.

Witte, J.F. (1997). Achievement Effects of the Milwaukee Voucher Program. Paper presented at the 1997 American Economics Association Annual Meeting: Madison [mimeo].

Witte, J.F., Thorn, C.A. \& Sterr, T. (1995). Fifth-Year Report: The Milwaukee Parental Choice 
Program. Report to the Wisconsin State Legislature. Madison: Department of Public Instruction.

Witte, J.F., Thorn, C.A., Pritchard, K. \& Claibourn, M. (1994). Fourth Year Report: Milwaukee Parental Choice Program. Report to the Wisconsin State Legislature. Madison: Department of Public Instruction.

Witte, J.F. \& Thorn, C.A.1996). Who Chooses? Voucher and Interdistrict Choice Programs in Milwaukee. American Journal of Education, 104, 186-216.

Wössmann, L. (2002). Schooling and the Quality of Human Capital. Kieler Studien 319. Kiel: Institut für Weltwirtschaft.

Wössmann, L. \& West, M.R. (2002). Class-Size Effects in School Systems Around the World: Evidence from Between-Grade Variation in TIMSS. Kiel Working Paper, 1099. Kiel: Institut für Weltwirtschaft.

Wolter, S.C. (2001). Bildungsfinanzierung zwischen Markt und Staat. Chur: Rüegger.

Worthington, A.C. (2001). An Empirical Survey of Frontier Efficiency Measurement Techniques in Education. Education Economics, 9, (3), 245-268.

\section{Efficacité interne des systèmes éducatifs}

\section{Résumé}

L'article examine les principaux facteurs qui influent sur l'efficacité des systèmes éducatifs, et les possibilités des les améliorer. A partir d'une analyse des intérêts personnels des groupes de participants aux systèmes éducatifs, l'auteur passe en revue les éléments qui peuvent déterminer l'efficacité des systèmes éducatifs; ceux-ci sont regroupés en trois domaines: les facteurs d'inputs et d'outputs analysés dans la perspective d'une fonction de production; les structures organisationnelles et les nouveaux modèles de financement éducatif, orientés vers une éducation plus concurrentielle et de marché.

Si l'on considère les résultats des recherches empiriques, une augmentation des ressources à elle seule a peu d'influence sur les résultats des élèves. La réaffectation des ressources existantes en faveur des moyens d'enseignement ou l'introduction d'un système de rémunération à la performance, et surtout des mesures structurelles, seraient plus prometteuses. Il en va de même des procédures centralisées d'examens qui semblent aboutir à des performances scolaires significativement meilleures. C'est aussi le cas de la décentralisation des responsabilités de gestion et du personnel. Le transfert total des responsabilités liées à la gestion des budgets et des installations entraîne plutôt une baisse des résultats des élèves, étant donné que les ressources sont plus particulièrement allouées en fonction des intérêts du corps enseignant.

Les effets des chèques éducatifs et autres instruments de financement plus orientés vers le marché, sont fortement controversés. Si l'on se réfere aux travaux empiriques portant sur de tels modèles, l'augmentation de performances des élèves est moins marquée que ne le prétendent de nombreux partisans du 
chèque. Néanmoins, la plupart des études concluent à une relation faiblement positive entre concurrence, chèques éducatifs et résultats des élèves. Cette relation peut être améliorée par des réformes structurelles affectant les systèmes éducatifs et pouvant conduire à des adaptations apportées aux modèles de chèques (mécanismes incitatifs différenciés en fonction de facteurs socio-économiques et individuels) ou à des mesures pour conseiller et orienter les usagers. Une réforme structurelle exhaustive et bien conçue incitera tous les participants au système éducatif à accroître la performance dudit système. Si l'on met en oeuvre des solutions partielles, il faudra alors s'attendre à des gains d'efficacité moindres.

\section{Efficacia interna dei sistemi educativi}

\section{Riassunto}

L'articolo discute i principali fattori che influenzano i sistemi educativi e le possibilità del loro miglioramento. Muovendo dall'analisi degli interessi specifici dei gruppi direttamente coinvolti, l'autore passa in rassegna i diversi elementi collocandoli in tre ambiti: i fattori di input e output visti nell'ottica di una funzione di produzione, le strutture organizzative e i nuovi modelli di finanziamento che accentuano l'orientamento verso una maggiore competitività e verso il mercato.

Le ricerche empiriche disponibili sembrano indicare che il solo incremento delle risorse abbia un'incidenza lieve sulle prestazioni degli allievi. Maggior successo promettono misure di riallocazione delle risorse a favore dei mezzi d'insegnamento, oppure un sistema di remunerazione basato sul merito e soprattutto misure di carattere strutturale. Così ad es. dispositivi di valutazione centralizzati comportano risultati significativamente migliori. Ciò vale anche per la decentralizzazione delle competenze relative alla gestione e al personale. L'attribuzione della completa responsabilità budgetaria induce invece piuttosto un calo delle prestazioni degli allievi, in quanto le risorse vengono utilizzate piuttosto a favore degli interessi degli insegnanti.

Molto controversi sono gli effetti dei cosiddetti buoni di formazione e di altre misure tese ad incrementare la concorrenza. Le ricerche empiriche mostrano come i risultati ottenuti dagli allievi sono inferiori a quanto preteso dai promotori di tali modelli. Tuttavia la maggior parte degli studi approda ad esiti leggermente positivi che possono essere migliorati con ulteriori misure strutturali. Di queste misure fanno parte tanto quelle attinenti alle modifiche del contesto strutturale quanto quelle riguardanti dispositivi di sostegno differenziati e in particolare l'assistenza e l'informazione. Una riforma globale e strutturata su queste basi può stimolare gli attori coinvolti a raggiungere risultati migliori. Se ci si limita a misure parziali, i risultati saranno inferiori. 


\title{
Internal Efficiency of Educational Systems
}

\begin{abstract}
The paper examines both the major factors determining and affecting the efficiency of educational systems and the opportunities for improving them. Starting from an analysis of individual interests of those who are the most concerned with and affected by the education system, connections between efficiency in the education system will be researched. The evidence for efficiency measures is investigated in three important areas of interest: namely input and output factors based on an educational production function approach, organisational structures, and new approaches to financing education, which are directed at more competitive and market-like education.

However, when one considers empirical evidence than it seems that increasing resources has only a small effect on student achievement. But there is some evidence that a reallocation of existing resources towards teaching and learning materials and performance-related teacher salaries will have a positive effect in this respect. The same positive effect on student achievement applies to centralized examination procedures, as well as more school autonomy concerning process and personnel. Autonomous budget responsibility for educational institutions leads to lower student achievement because resources are particularly used to serve the interests of the teachers.

The effects of educational coupons and other more market-oriented financing instruments are highly controversial. Looking at empirical studies, the positive effects on students are weaker than is stated by many proponents. Nonetheless, most studies conclude that there seems to be a positive relationship between competition and coupons and student's academic performance. However, the strongest impact of new financing models can be expected when it is combined with structural reforms, e.g. centralized exams. The social problems that are reported in connection with such reforms can be avoided by appropriate construction of the coupon systems (differentiating them according to socio-economic and individual factors) in combination with advice and information. Such a comprehensive and well thought out reform would increase the incentives for all those involved to improve the performance of the educational system. When only partial solutions are applied, gains in efficiency will be less if some small steps are not taken.
\end{abstract}


T h e m a 